PoS $\quad \begin{aligned} & \text { PROCEEDINGS } \\ & \text { OF SCIENCE }\end{aligned}$

\title{
Renormdynamics, coupling constant unification and universal properties of the multiparticle production
}

\author{
Nugzar Makhaldiani* \\ JINR \\ E-mail: mnvejinr.ru
}

After short introduction, we consider different aspects of the renormdynamics. Than scaling functions of the multiparticle production processes and corresponding stochastic dynamics are considered.

XXI International Baldin Seminar on High Energy Physics Problems September 10-15, 2012

JINR, Dubna, Russia

${ }^{*}$ Speaker. 


\section{Introduction}

In the Universe, matter has manly two geometric structures, homogeneous, [Weinberg, 1972] and hierarchical, [Okun, 1982] .

The homogeneous structures are naturally described by real numbers with an infinite number of digits in the fractional part and usual archimedean metrics. The hierarchical structures are described with p-adic numbers with an infinite number of digits in the integer part and nonarchimedean metrics, [Koblitz, 1977].

A discrete, finite, regularized, version of the homogenous structures are homogeneous lattices with constant steps and distance rising as arithmetic progression. The discrete version of the hierarchical structures is hierarchical lattice-tree with scale rising in geometric progression.

There is an opinion that present day theoretical physics needs (almost) all mathematics, and the progress of modern mathematics is stimulated by fundamental problems of theoretical physics.

\section{Quantum field theory and Fractal calculus - Universal language of fundamental physics}

In QFT existence of a given theory means, that we can control its behavior at some scales (short or large distances) by renormalization theory [Collins, 1984].

If the theory exists, than we want to solve it, which means to determine what happens on other (large or short) scales. This is the problem (and content) of Renormdynamics.

The result of the Renormdynamics, the solution of its discrete or continual motion equations, is the effective QFT on a given scale (different from the initial one).

We can invent scale variable $\lambda$ and consider QFT on $D+1+1$ dimensional space-time-scale. For the scale variable $\lambda \in(0,1]$ it is natural to consider $q$-discretization, $0<q<1, \lambda_{n}=q^{n}, n=$ $0,1,2, \ldots$ and $p$ - adic, nonarchimedian metric, with $q^{-1}=p$ - prime integer number.

The field variable $\varphi(x, t, \lambda)$ is complex function of the real, $\mathrm{x}, \mathrm{t}$, and $\mathrm{p}$ - adic, $\lambda$, variables. The solution of the UV renormdynamic problem means, to find evolution from finite to small scales with respect to the scale time $\tau=\ln \lambda / \lambda_{0} \in(0,-\infty)$. Solution of the IR renormdynamic problem means to find evolution from finite to the large scales, $\tau=\ln \lambda / \lambda_{0} \in(0, \infty)$.

This evolution is determined by Renormdynamic motion equations with respect to the scaletime.

As a concrete model, we take a relativistic scalar field model with lagrangian

$$
L=\frac{1}{2} \partial_{\mu} \varphi \partial^{\mu} \varphi-\frac{m^{2}}{2} \varphi^{2}-\frac{g}{n} \varphi^{n}, \mu=0,1, \ldots, D-1
$$

The mass dimension of the coupling constant is

$$
[g]=d_{g}=D-n \frac{D-2}{2}=D+n-\frac{n D}{2} .
$$

In the case

$$
n=\frac{2 D}{D-2}=2+\frac{4}{D-2}=2+\varepsilon(D)
$$




$$
D=\frac{2 n}{n-2}=2+\frac{4}{n-2}=2+\varepsilon(n)
$$

the coupling constant $\mathrm{g}$ is dimensionless, and the model is renormalizable.

We take the euklidean form of the QFT which unifies quantum and statistical physics problems. In the case of the QFT, we can return (in)to minkowsky space by transformation: $p_{D}=i p_{0}, x_{D}=$ $-i x_{0}$.

The main objects of the theory are Green functions - correlation functions - correlators,

$$
\begin{aligned}
& G_{m}\left(x_{1}, x_{2}, \ldots, x_{m}\right)=<\varphi\left(x_{1}\right) \varphi\left(x_{2}\right) \ldots \varphi\left(x_{m}\right)> \\
& =Z_{0}^{-1} \int d \varphi(x) \varphi\left(x_{1}\right) \varphi\left(x_{2}\right) \ldots \varphi\left(x_{m}\right) e^{-S(\varphi)}
\end{aligned}
$$

where $d \varphi$ is an invariant measure,

$$
d(\varphi+a)=d \varphi
$$

For gaussian actions,

$$
S=S_{2}=\frac{1}{2} \int d x d y \varphi(x) A(x, y) \varphi(y)=\varphi \cdot A \cdot \varphi
$$

the QFT is solvable,

$$
\begin{aligned}
& G_{m}\left(x_{1}, \ldots, x_{m}\right)=\left.\frac{\delta^{m}}{\delta J\left(x_{1}\right) \ldots J\left(x_{m}\right)} \ln Z_{J}\right|_{J=0}, \\
& Z_{J}=\int d \varphi e^{-S_{2}+J \cdot \varphi}=\exp \left(\frac{1}{2} \int d x d y J(x) A^{-1}(x, y) J(y)\right) \\
& =\exp \left(\frac{1}{2} J \cdot A^{-1} \cdot J\right)
\end{aligned}
$$

This solution is based on the solution of the linear motion equations with sources

$$
\int A(x, y) \varphi(y) d y=J(x)
$$

Nontrivial problem is to calculate correlators for non gaussian QFT.

\subsection{Effective action}

Generating functional for connected correlators is

$$
F(J)=\ln Z_{J}, \frac{\delta F(J)}{\delta J(x)}=\frac{1}{Z_{J}} \frac{\delta Z_{J}}{\delta J(x)} \equiv<\varphi(x)>_{J} \equiv \phi(x)-
$$

is observable value of the field, generated by source $J$. We have

$$
\left.\frac{\delta}{\delta J}(F(J)-J \cdot \phi)\right|_{\phi=c o n s t}=0
$$

so

$$
J \cdot \phi-F(J)=S_{q}(\phi)=S(\phi)+R(\phi)
$$




$$
\begin{aligned}
& =\sum_{n \geq 1} \frac{1}{n !} \int d x_{1} d x_{2} \ldots d x_{n} \Gamma_{n}\left(x_{1}, x_{2}, \ldots, x_{n}\right) \phi\left(x_{1}\right) \phi\left(x_{2}\right) \ldots \phi\left(x_{n}\right), \\
& \frac{\delta S_{q}}{\delta \phi(x)}=J(x) ; \frac{\delta^{2} S_{q}}{\delta \phi\left(x_{1}\right) \delta \phi\left(x_{2}\right)}=\frac{\delta J\left(x_{2}\right)}{\delta \phi\left(x_{1}\right)}=\frac{\delta J\left(x_{1}\right)}{\delta \phi\left(x_{2}\right)}=\Gamma_{2}\left(x_{1}, x_{2}\right)
\end{aligned}
$$

$R(\phi)$ - is quantum corrections to the classical action.

The connected part of the two point correlator - propagator, is

$$
\begin{aligned}
& <\varphi\left(x_{1}\right) \varphi\left(x_{2}\right)>_{c}=<\varphi\left(x_{1}\right) \varphi\left(x_{2}\right)>-<\varphi\left(x_{1}\right)><\varphi\left(x_{2}\right)> \\
& =\frac{1}{Z(J)} \frac{\delta^{2} Z(J)}{\delta J\left(x_{1}\right) \delta J\left(x_{2}\right)}-\frac{1}{Z(J)} \frac{\delta Z(J)}{\delta J\left(x_{1}\right)} \frac{1}{Z(J)} \frac{\delta Z(J)}{\delta J\left(x_{2}\right)}=\Gamma_{2}\left(x_{1}, x_{2}\right)
\end{aligned}
$$

\section{2 p-adic convergence of perturbative series}

Perturbative series have the following qualitative form

$$
\begin{aligned}
& f(g)=f_{0}+f_{1} g+\ldots+f_{n} g^{n}+\ldots, f_{n}=n ! P(n) \\
& f(x)=\sum_{n \geq 0} P(n) n ! x^{n}=P(\delta) \Gamma(1+\delta) \frac{1}{1-x}, \delta=x \frac{d}{d x}
\end{aligned}
$$

In the usual sense these series are divergent, but with proper nomalization of the expansion parametre $g$, the coefficients of the series are rational numbers and if experimental dates indicates for some rational value for $g$, e.g. in QED

$$
g=\frac{e^{2}}{4 \pi}=\frac{1}{137.0 \ldots}
$$

then we can take corresponding prime number and consider p-adic convergence of the series. In the case of QED, we have

$$
\begin{aligned}
& f(g)=\sum f_{n} p^{-n}, f_{n}=n ! P(n), p=137 \\
& |f|_{p} \leq \sum\left|f_{n}\right|_{p} p^{n}
\end{aligned}
$$

In the Yukawa theory of strong interactions (see e.g. [Bogoliubov, Shirkov, 1959]), we take $g=13$,

$$
\begin{aligned}
& f(g)=\sum f_{n} p^{n}, f_{n}=n ! P(n), p=13, \\
& |f|_{p} \leq \sum\left|f_{n}\right|_{p} p^{-n}<\frac{1}{1-p^{-1}}
\end{aligned}
$$

So, the series is convergent. If the limit is rational number, we consider it as an observable value of the corresponding physical quantity. Note also, that the inverse coupling expansions, e.g. in lattice(gauge) theories,

$$
f(\beta)=\sum r_{n} \beta^{n},
$$

are also p-adically convergent for $\beta=p^{k}$. We can take the following scenery. We fix coupling constants and masses, e.g in QED or QCD, in low order perturbative expansions. Than put the models on lattice and calculate observable quantities as inverse coupling expansions, e.g.

$$
f(\alpha)=\sum r_{n} \alpha^{-n}
$$




$$
\alpha_{Q E D}(0)=1 / 137 ; \alpha_{Q C D}\left(m_{Z}\right)=0.11 \ldots=1 / 3^{2}
$$

In MSSM (see [Muehlleitner, CALC 2012], [Kazakov, 2004]) coupling constants of the SM unifies at $\alpha_{u}^{-1}=26.3 \pm 1.9 \pm 1$.

So,

$$
23.4<\alpha_{u}^{-1}<29.2
$$

Question: how many primes are in this interval?

$$
24,25,26,27,28,29
$$

Only one!

Proposal: take the value $\alpha_{u}^{-1}=29.0 \ldots$ which will be two orders of magnitude more precise prediction and find the consequences for the $S M$ scale observables.

Remind that for low energy limit of the fine structure constant $\alpha, \alpha^{-1}=137.036 \ldots$

\subsection{The Goldberger-Treiman relation and the pion-nucleon coupling constant}

The Goldberger-Treiman relation (GTR) [Goldberger, Treiman, 1958] plays an important role in theoretical hadronic and nuclear physics. GTR relates the Meson-Nucleon coupling constants to the axial-vector coupling constant in $\beta$-decay:

$$
m_{N} g_{A}(0)=f_{\pi} g_{\pi N}
$$

where $m_{N}$ is the nucleon mass, $g_{A}(0)$ is the axial-vector coupling constant in nucleon $\beta$-decay at vanishing momentum transfer, $f_{\pi}$ is the $\pi$ decay constant and $g_{\pi N}$ is the $\pi-N$ coupling constant.

Since the days when the Goldberger-Treiman relation was discovered, the value of $g_{A}$ has increased considerably. Also, $f_{\pi}$ decreased a little, on account of radiative corrections. The main source of uncertainty is $g_{\pi N}$.

If we take

$$
\alpha_{\pi N}=\frac{g_{\pi N}^{2}}{4 \pi}=13, g_{\pi N}=12.78
$$

experimental value for $f_{\pi}$ from pion decay

$$
f_{\pi}=\frac{130}{\sqrt{2}}=91.9 \mathrm{MeV},
$$

Neutron mass,

$$
m_{N}=940 \mathrm{MeV}
$$

from (2.2]), we find

$$
g_{A}(0)=\frac{f_{\pi} g_{\pi N}}{m_{N}}=\frac{91.9 \times \sqrt{52 \pi}}{940}=1.2496 \simeq 1.25=\frac{5}{4},
$$

which coincides with the experimental value from $\beta$-decay

$$
g_{A}(0)=1.25
$$

So, we can say that using GTR we measured the pion-nucleon fine structure constant and find the value

$$
\alpha_{\pi N}=\frac{g_{\pi N}^{2}}{4 \pi}=13
$$




\subsection{A model of pion-nucleon interaction}

In a $\pi \rho N$ model of pion-nucleon interaction [Di Giacomo, Paffuti, Rossi, 1992]

$$
L_{\pi N}=g\left(\bar{N} \gamma_{\mu} t^{a} N+i \varepsilon^{a b c} \pi^{b} \partial_{\mu} \pi^{c}\right) \rho^{\mu a},
$$

pion interacts with nucleon through the exchange of the vector meson $\rho\left(m_{\rho}=750 \mathrm{MeV}, \mathrm{T}=1\right)$, the amplitude of $\rho^{0} \rightarrow \pi^{+} \pi^{-}$decay is

$$
M=g \varepsilon^{\mu}\left(k_{\pi^{-}}-k_{\pi^{+}}\right) \mu,
$$

the decay width is

$$
\Gamma=\frac{1}{2 m_{\rho}}|M|^{2}\left(1-\frac{4 m_{\pi}^{2}}{m_{\rho}^{2}}\right)^{\frac{1}{2}} \frac{1}{8 \pi}=\frac{g^{2}}{48 \pi} m_{\rho}\left(1-\frac{4 m_{\pi}^{2}}{m_{\rho}^{2}}\right)^{\frac{3}{2}}
$$

and for fine structure coupling constant we have

$$
\alpha_{\pi \rho N}=\frac{g^{2}}{4 \pi}=\frac{\Gamma}{m_{\rho}} \frac{12}{\left(1-\frac{4 m_{\pi}^{2}}{m_{\rho}^{2}}\right)^{\frac{3}{2}}}=\frac{12 .}{5\left(1-\frac{4 \times 14^{2}}{75^{2}}\right)^{\frac{3}{2}}}=3.006=3.0 .
$$

for $\Gamma=\Gamma_{\rho \pi \pi}=150 \mathrm{MeV}, m_{\pi}=140 \mathrm{MeV}, m_{\rho}=750 \mathrm{MeV}$. So, in this strong coupling model the expansion parameter is a prime number, $\alpha_{g}=3$.

\section{Neutral pion to two photon decay}

After integrating out all heavy and trapped particles, we would expect the effective Lagrangian for

$$
\pi^{0} \rightarrow 2 \gamma
$$

to be given by the unique gauge and Lorentz-invariant term with no more than two derivatives:

$$
L_{\pi \gamma \gamma}=g \pi^{0} \varepsilon^{\mu \nu \rho \sigma} F_{\mu \nu} F_{\rho \sigma}
$$

where $g$ is an unknown constant with the mass dimension $m^{-1}$.

The rate for $\pi^{0} \rightarrow 2 \gamma$ is

$$
\Gamma\left(\pi^{0} \rightarrow 2 \gamma\right)=\frac{g^{2} m_{\pi}^{3}}{\pi}
$$

One might naively expect $g$ to be of order

$$
g=\frac{a^{2}}{F_{\pi}}, a=\frac{e}{4 \pi}
$$

where $F_{\pi}=190 \mathrm{MeV}$ is used as a typical strong interaction mass scale.

In 1949, using the pre-QCD theory of pions and nuclons with interaction lagrangian

$$
L_{\pi N N}=i g_{\pi N} \pi^{a} \bar{N} 2 t^{a} \gamma_{5} N
$$


Steinberger calculated the contribution to $g$ from triangle graphs with a single proton loop

$$
g=\frac{e^{2} g_{\pi N}}{32 \pi^{2} m_{N}}=a^{2} \frac{g_{\pi N}}{2 m_{N}}, a=\frac{e}{4 \pi} .
$$

From Goldberger-Treiman relation we have

$$
\frac{g_{\pi N}}{2 m_{N}}=\frac{g_{A}}{2 f_{\pi}}
$$

so,

$$
g=\frac{a^{2}}{F_{\pi}} g_{A}, g_{A}=1.25, F_{\pi}=2 f_{\pi}=184 M e V
$$

Using

$$
\begin{gathered}
g=\frac{a^{2}}{F_{\pi}}, a=\frac{e}{4 \pi}, F_{\pi}=190 \mathrm{MeV} \\
\Gamma\left(\pi^{0} \rightarrow 2 \gamma\right)=\frac{g^{2} m_{\pi}^{3}}{\pi}=\frac{a^{4} m_{\pi}^{3}}{\pi F_{\pi}^{2}}=\frac{\alpha^{2} m_{\pi}^{3}}{16 \pi^{3} F_{\pi}^{2}}=1.0 \times 10^{16} \mathrm{~s}^{-1}
\end{gathered}
$$

The observed rate is

$$
\Gamma\left(\pi^{0} \rightarrow 2 \gamma\right)_{\exp }=(1.19 \pm 0.08) \times 10^{16} s^{-1},
$$

which is in good agreement with the estimation.

\section{Sum rules}

Much of the hadronic dynamics at low energies is determined by nucleon and meson exchanges while the intermediate energy region is dominated by various resonances. Chiral symmetry is an important physical constraint that governs the meson-nucleon interactions at low energies and unitarity is an essential property at intermediate energies. A natural link between the low and intermediate energy regions is provided by various sum rules.

\subsection{Gerasimov, Drell-Hearn sum rules}

The Gerasimov, Drell-Hearn (GDH) sum rule [Gerasimov, 1965], [Drell, Hearn, 1966]

$$
\frac{\pi^{2} \alpha}{m^{2} s} k^{2}=\int_{-\infty}^{\infty} d t[\sigma(t, 1+s)-\sigma(t, 1-s)]
$$

relates the anomalous magnetic moment $k$ of a particle with spin $s$ and mass $m$ to the integral of the difference of polarized total photo-absorption cross-sections $\sigma(t, 1 \pm s)$ for total helicity $(1 \pm s)$ and energy $\omega=\omega_{0} e^{t}$. The anomalous magnetic moment is $k=(g-2) s$, i.e., the deviation of the gyromagnetic ratio $g$ from its natural value of 2 for any spin [Ferrara, Porrati, Telegdi, 1992]. In the case of the proton, e.g., $s=1 / 2, k=1.79$ 
It is interesting also to consider high values of $s$ for high spin nuclei, magnetics, string and extended particle models. Note that, for $s=0$, both sides of the relation is zero.

Let us present the integral from right-hand side as

$$
\begin{aligned}
& \int_{-\infty}^{\infty} d t \sum_{n=-s+1}^{s} A(t, n)=\sum_{n=-s+1}^{s} \int_{-\infty}^{\infty} d t A(t, n)=\sum_{n=-s+1}^{s} a(n), \\
& A(t, n)=\sigma(t, 1+n)-\sigma(t, n), a(n)=\int_{-\infty}^{\infty} d t A(t, n)
\end{aligned}
$$

One of the solutions of the GDH relation considered as an integral equation is

$$
\begin{aligned}
& a(n)=a=\frac{\pi^{2} \alpha}{2 m^{2}}(g-2)^{2}, \\
& b(n+1)=b(n)+a, b(n)=\int_{-\infty}^{\infty} d t \sigma(t, n)
\end{aligned}
$$

\subsection{Adler-Weisberger sum rule}

The Adler-Weisberger sum rule (AWS) [Adler, 1965], [Weisberger, 1966, "Adler, Dashen, 1965], [Treiman, Witten, Jackiw, Zumino, 1985], [Weinberg, 1996] relates integrals over the total cross section to the scattering amplitude calculated at a subthreshold kinematic point

$$
\begin{gathered}
1-g_{A}^{-2}=\frac{2 m_{N}^{2}}{\pi g_{\pi N}^{2}} \int_{0}^{\infty} d t\left[\sigma^{\pi^{+} N}(t)-\sigma^{\pi^{-} N}(t)\right] \\
=\sin ^{2} \theta=(3 / 5)^{2}
\end{gathered}
$$

where $\left.\sigma^{\pi^{ \pm} N}(t)\right)$ are the pion-nucleon cross sections at the energy $\omega=m_{\pi} e^{t}$. For the value of the axial coupling constant $g_{A}=5 / 4=1 / \cos \theta$, on the right-hand side of the AWS, we will have $\sin ^{2} \theta, \sin \theta=3 / 5, \theta=0.6435011087932845 * 180 / P i=36.86989764584403 \simeq 37^{\circ}$

Note that, we can define the pion decay constant from the Goldberger-Treiman relation

$$
f_{\pi}=\frac{g_{A} m_{N}}{g_{\pi N}}
$$

and put the AWS in the form

$$
g_{A}^{2}=1+\frac{2 f_{\pi}^{2}}{\pi} \int_{0}^{\infty} d t\left[\sigma^{\pi^{+} N}(t)-\sigma^{\pi^{-} N}(t)\right]
$$

\section{Real, $p$ - adic and $q$ - uantum fractal calculus}

Every (good) school boy/girl knows what is

$$
\frac{d^{n}}{d x^{n}}=\partial^{n}=(\partial)^{n},
$$

but what is its following extension

$$
\frac{d^{\alpha}}{d x^{\alpha}}=\partial^{\alpha}, \quad \alpha \in \Re ?
$$




\subsection{Euler, ... Liouville, ... Holmgren, ...}

Let us consider the integer derivatives of the monomials

$$
\begin{aligned}
\frac{d^{n}}{d x^{n}} x^{m} & =m(m-1) \ldots(m-(n-1)) x^{m-n}, \quad n \leq m, \\
& =\frac{\Gamma(m+1)}{\Gamma(m+1-n)} x^{m-n} .
\end{aligned}
$$

L.Euler (1707 - 1783) invented the following definition of the fractal derivatives,

$$
\frac{d^{\alpha}}{d x^{\alpha}} x^{\beta}=\frac{\Gamma(\beta+1)}{\Gamma(\beta+1-\alpha)} x^{\beta-\alpha} .
$$

J.Liouville (1809-1882) takes exponents as a base functions,

$$
\frac{d^{\alpha}}{d x^{\alpha}} e^{a x}=a^{\alpha} e^{a x}
$$

The following Cauchy formula

$$
I_{0, x}^{n} f=\int_{0}^{x} d x_{n} \int_{0}^{x_{n-1}} d x_{n-2} \ldots \int_{0}^{x_{2}} d x_{1} f\left(x_{1}\right)=\frac{1}{\Gamma(n)} \int_{0}^{x} d y(x-y)^{n-1} f(y)
$$

permits analytic extension from integer $n$ to complex $\alpha$,

$$
I_{0, x}^{\alpha} f=\frac{1}{\Gamma(\alpha)} \int_{0}^{x} d y(x-y)^{\alpha-1} f(y)
$$

J.H. Holmgren invented (in 1863) the following integral transformation,

$$
D_{c, x}^{-\alpha} f=\frac{1}{\Gamma(\alpha)} \int_{c}^{x}|x-t|^{\alpha-1} f(t) d t .
$$

It is easy to show that

$$
\begin{aligned}
D_{c, x}^{-\alpha} x^{m} & =\frac{\Gamma(m+1)}{\Gamma(m+1+\alpha)}\left(x^{m+\alpha}-c^{m+\alpha}\right), \\
D_{c, x}^{-\alpha} e^{a x} & =a^{-\alpha}\left(e^{a x}-e^{a c}\right),
\end{aligned}
$$

so, $c=0$, when $m+\alpha \geq 0$, in Holmgren's definition of the fractal calculus, corresponds to the Euler's definition, and $c=-\infty$, when $a>0$, corresponds to the Liouville's definition.

Holmgren's definition of the fractal calculus reduce to the Euler's definition for finite $c$, and to the Liouvill's definition for $c=\infty$,

$$
\begin{aligned}
& D_{c, x}^{-\alpha} f=D_{0, x}^{-\alpha} f-D_{0, c}^{-\alpha} f, \\
& D_{\infty, x}^{-\alpha} f=D_{-\infty, x}^{-\alpha} f-D_{-\infty, \infty}^{-\alpha} f .
\end{aligned}
$$

We considered the following modification of the $c=0$ case [Makhaldiani, 2003],

$$
D_{0, x}^{-\alpha} f=\frac{|x|^{\alpha}}{\Gamma(\alpha)} \int_{0}^{1}|1-t|^{\alpha-1} f(x t) d t,=\frac{|x|^{\alpha}}{\Gamma(\alpha)} B(\alpha, \partial x) f(x)
$$




$$
=|x|^{\alpha} \frac{\Gamma(\partial x)}{\Gamma(\alpha+\partial x)} f(x), f(x t)=t^{\frac{d}{d x}} f(x) .
$$

As an example, consider Euler B-function,

$$
B(\alpha, \beta)=\int_{0}^{1} d x|1-x|^{\alpha-1}|x|^{\beta-1}=\Gamma(\alpha) \Gamma(\beta) D_{01}^{-\alpha} D_{0 x}^{1-\beta} 1=\frac{\Gamma(\alpha) \Gamma(\beta)}{\Gamma(\alpha+\beta)}
$$

We can define also FC as

$$
D^{\alpha} f=\left(D^{-\alpha}\right)^{-1} f=\frac{\Gamma(\partial x+\alpha)}{\Gamma(\partial x)}\left(|x|^{-\alpha} f\right), \partial x=\delta+1, \delta=x \partial
$$

For the Liouville's case,

$$
\begin{gathered}
D_{-\infty, x}^{\alpha} f=\left(D_{-\infty, x}\right)^{\alpha} f=\left(\partial_{x}\right)^{\alpha} f \\
\partial_{x}^{-\alpha} f=\frac{1}{\Gamma(\alpha)} \int_{0}^{\infty} d t t^{\alpha-1} e^{-t \partial_{x}} f(x)=\frac{1}{\Gamma(\alpha)} \int_{0}^{\infty} d t t^{\alpha-1} f(x-t) \\
=\frac{1}{\Gamma(\alpha)} \int_{-\infty}^{x} d t(x-t)^{\alpha-1} f(t)=D_{-\infty, x}^{-\alpha} f .
\end{gathered}
$$

The integrals can be calculated as

$$
D^{-n} f=\left(D^{-1}\right)^{n} f
$$

where

$$
D^{-1} f=x \frac{\Gamma(\partial x)}{\Gamma(1+\partial x)} f=x \frac{1}{\partial x} f=x(\partial x)^{-1} f=(\partial)^{-1} f=\int_{0}^{x} d t f(t) .
$$

Let us consider Weierstrass C.T.W. (1815 - 1897) fractal function

$$
f(t)=\sum_{n \geq 0} a^{n} e^{i\left(b^{n} t+\varphi_{n}\right)}, a<1, a b>1 .
$$

For fractals we have no integer derivatives,

$$
f^{(1)}(t)=i \sum(a b)^{n} e^{i\left(b^{n} t+\varphi_{n}\right)}=\infty
$$

but the fractal derivative,

$$
f^{(\alpha)}(t)=\sum\left(a b^{\alpha}\right)^{n} e^{i\left(b^{n} t+\pi \alpha / 2+\varphi_{n}\right)}
$$

when $a b^{\alpha}=a^{\prime}<1$, is another fractal (5.18).

Question: what if $a b=p$ is prime number? Can we define integer derivatives in this case? 


\section{$5.2 \mathrm{p}$ - adic fractal calculus}

p-adic analog of the fractal calculus (5.8),

$$
D_{x}^{-\alpha} f=\frac{1}{\Gamma_{p}(\alpha)} \int_{Q_{p}}|x-t|_{p}^{\alpha-1} f(t) d t
$$

where $f(x)$ is a complex function of the $\mathrm{p}$-adic variable $\mathrm{x}$, with $\mathrm{p}$-adic $\Gamma$-function

$$
\Gamma_{p}(\alpha)=\int_{Q_{p}} d t|t|_{p}^{\alpha-1} \chi(t)=\frac{1-p^{\alpha-1}}{1-p^{-\alpha}},
$$

was considered by V.S. Vladimirov [Dladimirov, 1988].

The following modification of p-adic FC is given in [Makhaldiani, 2003]

$$
\begin{aligned}
D_{x}^{-\alpha} f & =\frac{|x|_{p}^{\alpha}}{\Gamma_{p}(\alpha)} \int_{Q_{p}}|1-t|_{p}^{\alpha-1} f(x t) d t \\
& =|x|_{p}^{\alpha} \frac{\Gamma_{p}(\partial|x|)}{\Gamma_{p}(\alpha+\partial|x|)} f(x) .
\end{aligned}
$$

\subsection{Fractal qalculus}

The basic object of q-calculus [Gasper, Rahman, 1990] is q-derivative

$$
D_{q} f(x)=\frac{f(x)-f(q x)}{(1-q) x}=\frac{1-q^{x \partial}}{(1-q) x} f(x),
$$

where either $0<q<1$ or $1<q<\infty$. In the limit $q \rightarrow 1, D_{q} \rightarrow \partial_{x}$.

Now we define the fractal q-calculus,

$$
\begin{aligned}
& D_{q}^{\alpha} f(x)=\left(D_{q}\right)^{\alpha} f(x) \\
& =((1-q) x)^{-\alpha}\left(f(x)+\sum_{n \geq 1}(-1)^{n} \frac{\alpha(\alpha-1) \ldots(\alpha-n+1)}{n !} f\left(q^{n} x\right)\right) .
\end{aligned}
$$

For the case $\alpha=-1$, we obtain the integral

$$
D_{q}^{-1} f(x)=(1-q) x\left(1-q^{x \partial}\right)^{-1} f(x)=(1-q) x \sum_{n \geq 0} f\left(q^{n} x\right) .
$$

In the case of $1<q<\infty$, we can give a good analytic sense to these expressions for prime numbers $q=p=2,3,5, \ldots, 29, \ldots, 137, \ldots$ This is an algebra-analytic quantization of the q-calculus and corresponding physical models. Note also, that p-adic calculus is the natural tool for the physical models defined on the fractal( space)s like Bete lattice ( or Brua-Tits trees, in mathematical literature).

Note also a symmetric definition of the calculus

$$
D_{q s} f(x)=\frac{f\left(q^{-1} x\right)-f(q x)}{\left(q^{-1}-q\right) x} f(x) .
$$




\subsection{Fractal finite - difference calculus}

Usual finite difference calculus is based on the following (left) derivative operator

$$
D_{-} f(x)=\frac{f(x)-f(x-h)}{h}=\left(\frac{1-e^{-h \partial}}{h}\right) f(x) .
$$

We define corresponding fractal calculus as

$$
D_{-}^{\alpha} f(x)=\left(D_{-}\right)^{\alpha} f(x) .
$$

In the case of $\alpha=-1$, we have usual finite difference sum as regularization of the Riemann integral

$$
D_{-}^{-1} f(x)=h(f(x)+f(x-h)+f(x-2 h)+\ldots) .
$$

(I believe that) the fractal calculus (and geometry) are the proper language for the quantume (field) theories, and discrete versions of the fractal calculus are proper regularizations of the fractal calculus and field theories.

\subsection{Hypergeometric functions}

A hypergeometric series, in the most general sense, is a power series in which the ratio of successive coefficients indexed by $n$ is a rational function of $n$,

$$
f(x)=\sum_{n \geq 0} a_{n} x^{n}, a_{n+1}=R(n) a_{n}, R(n)=\frac{P(\alpha, n)}{Q(\beta, n)}
$$

so

$$
\begin{aligned}
& P(\alpha, \delta) f(x)=Q(\beta, \delta)(f(x)-f(0)) / x \\
& f(x)-f(0)=x R(\delta) f(x), f(x)=(1-x R(\delta))^{-1} f(0), \delta=x \partial_{x}
\end{aligned}
$$

Hypergeometric functions have many particular special functions as special cases, including many elementary functions, the Bessel functions, the incomplete gamma function, the error function, the elliptic integrals and the classical orthogonal polynomials, because the hypergeometric functions are solutions to the hypergeometric differential equation, which is a fairly general second-order ordinary differential equation.

In a generalization given by Eduard Heine ( 1821 - 1881 ), the ratio of successive terms, instead of being a rational function of $\mathrm{n}$, are considered to be a rational function of $q^{n}$

$$
\begin{aligned}
& f(x)=\sum_{n \geq 0} a_{n} x^{n}, a_{n+1}=R\left(q^{n}\right) a_{n}, R(n)=\frac{P\left(\alpha, q^{n}\right)}{Q\left(\beta, q^{n}\right)}, \\
& P\left(\alpha, q^{\delta}\right) f(x)=Q\left(\beta, q^{\delta}\right)(f(x)-f(0)) / x, \\
& f(x)-f(0)=x R\left(q^{\delta}\right) f(x), f(x)=\left(1-x R\left(q^{\delta}\right)\right)^{-1} f(0), \delta=x \partial_{x}
\end{aligned}
$$

Another generalization, the elliptic hypergeometric series, are those series where the ratio of terms is an elliptic function (a doubly periodic meromorphic function) of $n$.

There are a number of new definitions of hypergeometric series, by Aomoto, Gelfand and others; and applications for example to the combinatorics of arranging a number of hyperplanes in complex $\mathrm{N}$-space. 


\subsection{Hypergeometric field theory (HFT)}

Formal solutions for the the hypergeometric functions (5.32],5.33]), we put in the fieldtheoretic form,

$$
\begin{aligned}
& f(x)=G(x) f(0), \\
& G(x)=<\psi(x) \phi(0)>=\frac{\delta^{2} \ln Z}{\delta J(x) \delta I(0)}=(1-x R)^{-1}, \\
& Z=\int d \psi d \phi e^{-S+I \phi+J \psi}=e^{I(1-x R)^{-1} J}, \\
& S=\int \psi(1-x R) \phi=\int \psi(Q-x P) \varphi, \phi=Q \varphi .
\end{aligned}
$$

When we invent interaction terms, we obtain nontrivial HFT. In terms of the fundamental fields, $\psi, \varphi$, we have local field model.

\subsection{Lauricella Hypergeometric functions (LFs)}

For LFs (see, e.g. [Miller, 1977]), we find the following formulas [Makhaldiani, 201]

$$
\begin{aligned}
& F_{A}\left(a ; b_{1}, \ldots, b_{n} ; c_{1}, \ldots, c_{n} ; z_{1}, \ldots, z_{n}\right)=\frac{(a)_{\delta_{1}+\ldots+\delta_{n}}\left(b_{1}\right)_{\delta_{1} \ldots(}\left(b_{n}\right)_{\delta_{n}}}{\left(c_{1}\right)_{\delta_{1}} \ldots\left(c_{n}\right)_{\delta_{n}}} e^{z_{1}+\ldots+z_{n}} \\
& =\frac{(a)_{\delta_{1}+\ldots+\delta_{n}}}{\left(a_{1}\right)_{\delta_{1}} \ldots\left(a_{n}\right)_{\delta_{n}}} F\left(a_{1}, b_{1} ; c_{1} ; z_{1}\right) \ldots F\left(a_{n}, b_{n} ; c_{n} ; z_{n}\right) \\
& =T^{-1}(a) F^{n}=\Sigma_{m \geq 0} \frac{(a)_{m_{1}+\ldots+m_{n}}\left(b_{1}\right)_{m_{1}} \ldots\left(b_{n}\right)_{m_{n}}}{\left(c_{1}\right)_{m_{1}} \ldots\left(c_{n}\right)_{m_{n}}} \frac{z_{1}^{m_{1}}}{m_{1} !} \ldots \frac{z_{n}^{m_{n}}}{m_{n} !},\left|z_{1}\right|+\ldots+\left|z_{n}\right|<1 \text {; } \\
& F_{B}\left(a_{1}, \ldots, a_{n} ; b_{1}, \ldots, b_{n} ; c ; z_{1}, \ldots, z_{n}\right)=\frac{\left(a_{1}\right)_{\delta_{1}} \ldots\left(a_{n}\right)_{\delta_{n}}\left(b_{1}\right)_{\delta_{1}} \ldots\left(b_{n}\right)_{\delta_{n}}}{(c)_{\delta_{1}+\ldots+\delta_{n}}} e^{z_{1}+\ldots+z_{n}} \\
& =\frac{\left(c_{1}\right)_{\delta_{1}} \ldots\left(c_{n}\right)_{\delta_{n}}}{(c)_{\delta_{1}+\ldots+\delta_{n}}} F\left(a_{1}, b_{1} ; c_{1} ; z_{1}\right) \ldots F\left(a_{n}, b_{n} ; c_{n} ; z_{n}\right)=T(c) F^{n} \\
& =\Sigma_{m \geq 0} \frac{\left(a_{1}\right)_{m_{1}} \ldots\left(a_{n}\right)_{m_{n}}\left(b_{1}\right)_{m_{1}} \ldots\left(b_{n}\right)_{m_{n}}}{(c)_{m_{1}+\ldots+m_{n}}} \frac{z_{1}^{m_{1}}}{m_{1} !} \ldots \frac{z_{n}^{m_{n}}}{m_{n} !},\left|z_{1}\right|<1, \ldots,\left|z_{n}\right|<1 \text {; } \\
& F_{C}\left(a ; b ; c_{1}, \ldots, c_{n} ; z_{1}, \ldots, z_{n}\right)=\frac{(a)_{\delta_{1}+\ldots+\delta_{n}}(b)_{\delta_{1}+\ldots+\delta_{n}}}{\left(c_{1}\right)_{\delta_{1}} \ldots\left(c_{n}\right)_{\delta_{n}}} e^{z_{1}+\ldots+z_{n}} \\
& =\frac{(a)_{\delta_{1}+\ldots+\delta_{n}}(b)_{\delta_{1}+\ldots+\delta_{n}}}{\left(a_{1}\right)_{\delta_{1}} \ldots\left(a_{n}\right)_{\delta_{n}}\left(b_{1}\right)_{\delta_{1} \ldots\left(b_{n}\right)_{\delta_{n}}}} F\left(a_{1}, b_{1} ; c_{1} ; z_{1}\right) \ldots F\left(a_{n}, b_{n} ; c_{n} ; z_{n}\right) \\
& =T^{-1}(a) T^{-1}(b) F^{n}=T^{-1}(b) F_{A} \\
& =\Sigma_{m \geq 0} \frac{(a)_{m_{1}+\ldots+m_{n}}(b)_{m_{1}+\ldots+m_{n}}}{\left(c_{1}\right)_{m_{1}} \ldots\left(c_{n}\right)_{m_{n}}} \frac{z_{1}^{m_{1}}}{m_{1} !} \ldots \frac{z_{n}^{m_{n}}}{m_{n} !},\left|z_{1}\right|^{1 / 2}+\ldots+\left|z_{n}\right|^{1 / 2}<1 ; \\
& F_{D}\left(a ; b_{1}, \ldots, b_{n} ; c ; z_{1}, \ldots, z_{n}\right)=\frac{(a)_{\delta_{1}+\ldots+\delta_{n}}\left(b_{1}\right)_{\delta_{1}} \ldots\left(b_{n}\right)_{\delta_{n}}}{(c)_{\delta_{1}+\ldots \delta_{n}}} e^{z_{1}+\ldots+z_{n}} \\
& =\frac{(a)_{\delta_{1}+\ldots+\delta_{n}}\left(c_{1}\right)_{\delta_{1} \ldots\left(c_{n}\right)_{\delta_{n}}}}{\left(a_{1}\right)_{\delta_{1} \ldots\left(a_{n}\right)_{\delta_{n}}(c){ }_{\delta_{1}+\ldots \delta_{n}}}} F\left(a_{1}, b_{1} ; c_{1} ; z_{1}\right) \ldots F\left(a_{n}, b_{n} ; c_{n} ; z_{n}\right) \\
& =T^{-1}(a) T(c) F^{n}=T(c) F_{A}=T^{-1}(a) F_{B} \\
& =\Sigma_{m \geq 0} \frac{(a)_{m_{1}+\ldots+m_{n}}\left(b_{1}\right)_{m_{1}} \ldots\left(b_{n}\right)_{m_{n}}}{\left(c_{1}\right)_{m_{1}} \ldots\left(c_{n}\right)_{m_{n}}} \frac{z_{1}^{m_{1}}}{m_{1} !} \ldots \frac{z_{n}^{m_{n}}}{m_{n} !},\left|z_{1}\right|<1, \ldots,\left|z_{n}\right|<1 \text {. }
\end{aligned}
$$




\subsection{Lomidze $B_{n}$ function (LBn)}

In the paper ([Lomidze, 1994]) the following formula were proposed

$$
\begin{aligned}
& B_{n}\left(r_{0}, r_{1}, \ldots, r_{n}\right)=\operatorname{det}\left[x_{j}^{i-1} \int_{x_{j-1 / x_{j}}}^{1} u^{i-1}(1-u)^{r_{j}-1} \prod_{k=0, k \neq j}^{n}\left(\frac{x_{j} u-x_{k}}{x_{j}-x_{k}}\right)^{r_{k}-1} \operatorname{du}\right] / \operatorname{det}\left[x_{j}^{i-1}\right] \\
& =\frac{\Gamma\left(r_{0}\right) \Gamma\left(r_{1}\right) \ldots \Gamma\left(r_{n}\right)}{\Gamma\left(r_{o}+r_{1}+\ldots+r_{n}\right)}, 0=x_{0}<x_{1}<x_{2}<\ldots<x_{n}, n \geq 1
\end{aligned}
$$

Let us put the formula in the following factorized form

$$
\begin{aligned}
& L B_{n}(x, r) \equiv \operatorname{det}\left[x_{j}^{i-1} \int_{x_{j-1 / x_{j}}}^{1} d u u^{i+r_{0}-2}(1-u)^{r_{j}-1} \prod_{k=1, k \neq j}^{n}\left(\frac{x_{j} u-x_{k}}{x_{j}-x_{k}}\right)^{r_{k}-1}\right] \\
& =\operatorname{det} V_{n}(x) B_{n}(r), V_{n}(x)=\left[x_{j}^{i-1}\right], B_{n}(r)=\frac{\Gamma\left(r_{0}\right) \Gamma\left(r_{1}\right) \ldots \Gamma\left(r_{n}\right)}{\Gamma\left(r_{0}+r_{1}+\ldots+r_{n}\right)}
\end{aligned}
$$

Now, it is enough to proof this formula for general values of $x_{i}$ and particular values of $r_{i}$, e.g., $r_{i}=1$, and for general values of $r_{i}$ and particular values of $x_{i}$, e.g. $x_{i}=p^{i}, 1 \leq i \leq n$. In the case of $r_{i}=1$, right hand side of the formula is equal to the Vandermonde determinant divided by $n$ ! The left hand side is the determinant of the matrix with elements $A_{i j}=x_{j}^{i-1}\left(1-\left(x_{j-1} / x_{j}\right)^{i}\right) / i$

When we calculate determinant of this matrix, from the row $\mathrm{i}$, we factorize $1 / i, 2 \leq i \leq n$ which gives the $1 / n$ ! the rest matrix we calculate transforming the matrix to the form of the Vandermonde matrix.

This is the half way of the proof. Let us take the concrete values of $x_{i}=p^{i}, 1 \leq i \leq n$, where $p$ is positive integer and general complex values for $r_{i}, 0 \leq i \leq n$, and calculate both sides of the equality. For Vandermonde determinant we find for high values of $p$ the following asymptotic

$$
\operatorname{det} V=p^{N}, N=\sum_{k=2}^{n} k(k-1)=\frac{n\left(n^{2}-1\right)}{3}
$$

The matrix elements are

$$
\begin{aligned}
& B_{i j}=x_{j}^{i-1} \int_{x_{j-1 / x_{j}}}^{1} u^{i+r_{0}-2}(1-u)^{r_{j}-1} \prod_{k=1, k \neq j}^{n}\left(\frac{x_{j} u-x_{k}}{x_{j}-x_{k}}\right)^{r_{k}-1} d u \\
& =x_{j}^{i-1} \prod_{1 \leq k<j}\left(\frac{x_{j}}{x_{j}-x_{k}}\right)^{r_{k}-1} \prod_{j<k \leq n}\left(\frac{x_{k}}{x_{k}-x_{j}}\right)^{r_{k}-1} \int_{x_{j-1 / x_{j}}}^{1} u^{i+r_{0}-2}(1-u)^{r_{j}-1} \\
& \cdot \prod_{1 \leq k<j}\left(u-x_{k} / x_{j}\right)^{r_{k}-1} \prod_{j<k \leq n}\left(1-x_{j} / x_{k} u\right)^{r_{k}-1} d u \\
& =p^{(i-1) j} \int_{0}^{1} u^{i+r_{0}-2+\sum_{k=1}^{j-1}\left(r_{k}-1\right)}(1-u)^{r_{j}-1} d u \\
& =p^{(i-1) j} B\left(i+\sum_{k=0}^{j-1}\left(r_{k}-1\right), r_{j}\right)
\end{aligned}
$$

For $n=2$ we have

$$
\begin{aligned}
& B_{11}=\int_{0}^{1} u^{r_{0}-1}(1-u)^{r_{1}-1} d u=\frac{\Gamma\left(r_{0}\right) \Gamma\left(r_{1}\right)}{\Gamma\left(r_{0}+r_{1}\right)}, \\
& B_{22}=p^{2} \int_{0}^{1} u^{r_{0}+r_{1}-1}(1-u)^{r_{2}-1} d u=\frac{\Gamma\left(r_{0}+r_{1}\right) \Gamma\left(r_{2}\right)}{\Gamma\left(r_{0}+r_{1}+r_{2}\right)},
\end{aligned}
$$




$$
L B_{2} / V_{2}=B_{11} B_{22} / p^{2}=\frac{\Gamma\left(r_{0}\right) \Gamma\left(r_{1}\right) \Gamma\left(r_{2}\right)}{\Gamma\left(r_{0}+r_{1}+r_{2}\right)}
$$

For $n=3$,

$$
\begin{aligned}
& B_{11}=\int_{0}^{1} u^{r_{0}-1}(1-u)^{r_{1}-1}=\frac{\Gamma\left(r_{0}\right) \Gamma\left(r_{1}\right)}{\Gamma\left(r_{0}+r_{1}\right)}=B\left(r_{0}, r_{1}\right), \\
& B_{22}=p^{2} \int_{0}^{1} u^{r_{0}+r_{1}-1}(1-u)^{r_{2}-1}=p^{2} \frac{\Gamma\left(r_{0}+r_{1}\right) \Gamma\left(r_{2}\right)}{\Gamma\left(r_{0}+r_{1}+r_{2}\right)}, \\
& B_{33}=p^{6} \int_{0}^{1} u^{r_{0}+r_{1}+r_{2}-1}(1-u)^{r_{3}-1}=p^{6} \frac{\Gamma\left(r_{0}+r_{1}+r_{2}\right) \Gamma\left(r_{3}\right)}{\Gamma\left(r_{0}+r_{1}+r_{2}+r_{3}\right)} \\
& L B_{3} / V_{3}=B_{11} B_{22} B_{33} / p^{8}=\frac{\Gamma\left(r_{0}\right) \Gamma\left(r_{1}\right) \Gamma\left(r_{2}\right) \Gamma\left(r_{3}\right)}{\Gamma\left(r_{0}+r_{1}+r_{2}+r_{3}\right)}
\end{aligned}
$$

Now it is obvious the last step of the proof [Makhaldiani, 201]

$$
\begin{aligned}
& L B_{n}(x, r)=\operatorname{det} V_{n}(x) B\left(r_{0}, r_{1}\right) \ldots B\left(r_{0}+r_{1}+\ldots+r_{n-1}, r_{n}\right) \\
& =\operatorname{det}_{n}(x) B_{n}(r) \\
& V_{n}(x)=\left[x_{j}^{i-1}\right], B_{n}(r)=\frac{\Gamma\left(r_{0}\right) \Gamma\left(r_{1}\right) . . \Gamma\left(r_{n}\right)}{\Gamma\left(r_{0}+r_{1}+\ldots+r_{n}\right)}
\end{aligned}
$$

\subsection{Field theory applications of FC}

Let us consider the following action

$$
S=\frac{1}{2} \int_{Q_{v}} d x \Phi(x) D_{x}^{\alpha} \Phi, v=1,2,3,5, \ldots, 29, \ldots, 137, \ldots
$$

$Q_{1}$ is real number field, $Q_{p}, \mathrm{p}$ - prime, are p-adic number fields. In the momentum representation

$$
\begin{aligned}
& S=\frac{1}{2} \int_{Q_{v}} d u \tilde{\Phi}(-u)|u|_{v}^{\alpha} \tilde{\Phi}(u), \Phi(x)=\int_{Q_{v}} d u \chi_{v}(u x) \tilde{\Phi}(u), \\
& D^{-\alpha} \chi_{v}(u x)=|u|_{v}^{-\alpha} \chi_{v}(u x) .
\end{aligned}
$$

The statistical sum of the corresponding quantum theory is

$$
Z_{v}=\int d \Phi e^{-\frac{1}{2} \int \Phi D^{\alpha} \Phi}=d e t^{-1 / 2} D^{\alpha}=\left(\prod_{u}|u|_{v}\right)^{-\alpha / 2} .
$$

\subsection{String theory applications}

For (symmetrized, 4-tachyon) Veneziano amplitude we have (see, e.g. [Kaku, 2000])

$$
\begin{aligned}
& B_{s}(\alpha, \beta)=B(\alpha, \beta)+B(\beta, \gamma)+B(\gamma, \alpha)=\int_{-\infty}^{\infty} d x|1-x|^{\alpha-1}|x|^{\beta-1}, \\
& \alpha+\beta+\gamma=1
\end{aligned}
$$

For the p-adic Veneziano amplitude we take

$$
B_{p}(\alpha, \beta)=\int_{Q_{p}} d x|1-x|_{p}^{\alpha-1}|x|_{p}^{\beta-1}=\frac{\Gamma_{p}(\alpha) \Gamma_{p}(\beta)}{\Gamma_{p}(\alpha+\beta)}
$$


Now we obtain the N-tachyon amplitude using fractal calculus. We consider the dynamics of particle given by multicomponent generalization of the action (5.44), $\Phi \rightarrow x^{\mu}$.

For the closed trajectory of the particle passing through $N$ points, we have

$$
\begin{aligned}
& A\left(x_{1}, x_{2}, \ldots, x_{N}\right)=\int d t \int d t_{1} \ldots \int d t_{N} \delta\left(t-\Sigma t_{n}\right) \\
& v\left(x_{1}, t_{1} ; x_{2}, t_{2}\right) v\left(x_{2}, t_{2} ; x_{3}, t_{3}\right) \ldots v\left(x_{N}, t_{N} ; x_{1}, t_{1}\right) \\
& =\int d x(t) \Pi\left(\int d t_{n} \delta\left(x^{\mu}\left(t_{n}\right)-x_{n}^{\mu}\right)\right) \exp (-S[x(t)]) \\
& =\int \Pi\left(d k_{n}^{\mu} \chi\left(k_{n} x_{n}\right)\right) \tilde{A}(k),
\end{aligned}
$$

where

$$
\begin{aligned}
& \tilde{A}(k)=\int d x V\left(k_{1}\right) V\left(k_{2}\right) \ldots V\left(k_{N}\right) \exp (-S) \\
& V\left(k_{n}\right)=\int d t \chi\left(-k_{n} x(t)\right)
\end{aligned}
$$

is vertex function.

Motion equation

$$
D^{\alpha} x^{\mu}-i \Sigma k_{n}^{\mu} \delta\left(t-t_{n}\right)=0
$$

in the momentum representation

$$
|u|^{\alpha} \tilde{x}^{\mu}(u)-i \Sigma_{n} k_{n}^{\mu} \chi\left(-u t_{n}\right)=0
$$

have the solution

$$
\tilde{x}^{\mu}(u)=i \Sigma k_{n}^{\mu} \frac{\chi\left(-u t_{n}\right)}{|u|^{\alpha}}, u \neq 0
$$

the constraint

$$
\Sigma_{n} k_{n}=0
$$

and the zero $\bmod \tilde{x}_{n}^{\mu}(0)$, which is arbitrary. Integration in (5.49) with respect to this zero mod gives the constraint (5.54). On the solution of the equation (5.51)

$$
x^{\mu}(t)=i D_{t}^{-\alpha} \Sigma_{n} k_{n}^{\mu} \delta\left(t-t_{n}\right)=\frac{i}{\Gamma(\alpha)} \Sigma_{n} k_{n}^{\mu}\left|t-t_{n}\right|^{\alpha-1},
$$

the action (5.44) takes value

$$
\begin{aligned}
& S=-\frac{1}{\Gamma(\alpha)} \Sigma_{n<m} k_{n} k_{m}\left|t_{n}-t_{m}\right|^{\alpha-1}, \\
& \tilde{A}(k)=\int \Pi_{n=1}^{N} d t_{n} \exp (-S)
\end{aligned}
$$

In the limit, $\alpha \rightarrow 1$, for $p$-adic case we obtain

$$
x^{\mu}(t)=-i \frac{p-1}{p \ln p} \Sigma_{n} k_{n}^{\mu} \ln \left|t-t_{n}\right|,
$$




$$
\begin{aligned}
& S[x(t)]=\frac{p-1}{p \ln p} \Sigma_{n<m} k_{n} k_{m} \ln \left|t_{n}-t_{m}\right|, \\
& \tilde{A}(k)=\int \Pi_{n=1}^{N} d t_{n} \Pi_{n<m}\left|t_{n}-t_{m}\right|^{\frac{p-1}{p \ln } k_{n} k_{m}} .
\end{aligned}
$$

Now in the limit $p=q^{-1} \rightarrow 1$ we obtain the proper expressions of the real case

$$
\begin{aligned}
& x^{\mu}(t)=-i \Sigma_{n} k_{n}^{\mu} \ln \left|t-t_{n}\right|, \\
& S[x(t)]=\Sigma_{n<m} k_{n} k_{m} \ln \left|t_{n}-t_{m}\right|, \\
& \tilde{A}(k)=\int \Pi_{n=1}^{N} d t_{n} \Pi_{n<m}\left|t_{n}-t_{m}\right|_{n} k_{m} .
\end{aligned}
$$

By fractal calculus and vector generalization of the model (5.44), fundamental string amplitudes were obtained in [Makhaldiani, 1988].

\subsection{Renormdynamics of QCD}

QCD is the theory of the strong interactions with, as only inputs, one mass parameter for each quark species and the value of the QCD coupling constant at some energy or momentum scale in some renormalization scheme. This last free parameter of the theory can be fixed by $\Lambda_{Q C D}$, the energy scale used as the typical boundary condition for the integration of the Renormdynamic equation for the strong coupling constant. This is the parameter which expresses the scale of strong interactions, the only parameter in the limit of massless quarks. While the evolution of the coupling with the momentum scale is determined by the quantum corrections induced by the renormalization of the bare coupling and can be computed in perturbation theory, the strength itself of the interaction, given at any scale by the value of the renormalized coupling at this scale, or equivalently by $\Lambda_{Q C D}$, is one of the above mentioned parameters of the theory and has to be taken from experiment.

The RD equations play an important role in our understanding of Quantum Chromodynamics and the strong interactions. The beta function and the quarks mass anomalous dimension are among the most prominent objects for QCD RD equations. The calculation of the one-loop $\beta$-function in QCD has lead to the discovery of asymptotic freedom in this model and to the establishment of QCD as the theory of strong interactions [Gross, Wilczek, 1973, Politzer, 1973], t t Hooft, 1972].

The MS-scheme [t Hooft, 19722 belongs to the class of massless schemes where the $\beta$ function does not depend on masses of the theory and the first two coefficients of the $\beta$-function are scheme-independent.

The Lagrangian of QCD with massive quarks in the covariant gauge

$$
\begin{aligned}
& L=-\frac{1}{4} F_{\mu v}^{a} F^{a \mu v}+\bar{q}_{n}\left(i \gamma D-m_{n}\right) q_{n} \\
& -\frac{1}{2 \xi}(\partial A)^{2}+\partial^{\mu} \bar{c}^{a}\left(\partial_{\mu} c^{a}+g f^{a b c} A_{\mu}^{b} c^{c}\right) \\
& F_{\mu v}^{a}=\partial_{\mu} A_{v}^{a}-\partial_{v} A_{\mu}^{a}+g f^{a b c} A_{\mu}^{b} A_{v}^{c} \\
& \left(D_{\mu}\right)_{k l}=\delta_{k l} \partial_{\mu}-i g t_{k l}^{a} A_{\mu}^{a},
\end{aligned}
$$

$A_{\mu}^{a}, a=1, \ldots, N_{c}^{2}-1$ are gluon; $q_{n}, n=1, \ldots, n_{f}$ are quark; $c^{a}$ are ghost fields; $\xi$ is gauge parameter; $t^{a}$ are generators of fundamental representation and $f^{a b c}$ are structure constants of the Lie algebra

$$
\left[t^{a}, t^{b}\right]=i f^{a b c} t^{c},
$$


we will consider an arbitrary compact semi-simple Lie group G. For QCD, $G=S U\left(N_{c}\right), N_{c}=3$.

The RD equation for the coupling constant is

$$
\begin{aligned}
& \dot{a}=\beta(a)=-\beta_{2} a^{2}-\beta_{3} a^{3}-\beta_{4} a^{4}-\beta_{5} a^{5}+O\left(a^{6}\right), \\
& a=\frac{\alpha_{s}}{4 \pi}=\left(\frac{g}{4 \pi}\right)^{2} \\
& \int_{a_{0}}^{a} \frac{d a}{\beta(a)}=t-t_{0}=\ln \frac{\mu^{2}}{\mu_{0}^{2}},
\end{aligned}
$$

$\mu$ is the ' $t$ Hooft unit of mass, the renormalization point in the MS-scheme.

To calculate the $\beta$-function we need to calculate the renormalization constant $Z$ of the coupling constant, $a_{b}=Z a$, where $a_{b}$ is the bare (unrenormalized) charge.

The expression of the $\beta$-function can be obtained in the following way

$$
\begin{aligned}
& 0=d\left(a_{b} \mu^{2 \varepsilon}\right) / d t=\mu^{2 \varepsilon}\left(\varepsilon Z a+\frac{\partial(Z a)}{\partial a} \frac{d a}{d t}\right) \\
& \Rightarrow \frac{d a}{d t}=\beta(a, \varepsilon)=\frac{-\varepsilon Z a}{\frac{\partial(Z a)}{\partial a}}=-\varepsilon a+\beta(a), \\
& \beta(a)=a \frac{d}{d a}\left(a Z_{1}\right)
\end{aligned}
$$

where

$$
\beta(a, \varepsilon)=\frac{D-4}{2} a+\beta(a)
$$

is $D$-dimensional $\beta$-function and $Z_{1}$ is the residue of the first pole in $\varepsilon$ expansion

$$
Z(a, \varepsilon)=1+Z_{1} \varepsilon^{-1}+\ldots+Z_{n} \varepsilon^{-n}+\ldots
$$

Since $Z$ does not depend explicitly on $\mu$, the $\beta$-function is the same in all MS-like schemes, i.e. within the class of renormalization schemes which differ by the shift of the parameter $\mu$.

For quark anomalous dimension, RD equation is

$$
\begin{aligned}
& \dot{b}=\gamma(a)=-\gamma_{1} a-\gamma_{2} a^{2}-\gamma_{3} a^{3}-\gamma_{4} a^{4}+O\left(a^{5}\right), \\
& b=\ln m_{q}, \\
& b(t)=b_{0}+\int_{t_{0}}^{t} d t \gamma(a(t))=b_{0}+\int_{a_{0}}^{a} d a \gamma(a) / \beta(a) .
\end{aligned}
$$

To calculate the quark mass anomalous dimension $\gamma(g)$ we need to calculate the renormalization constant $Z_{m}$ of the quark mass $m_{b}=Z_{m} m, m_{b}$ is the bare (unrenormalized) quark mass. Than we find the function $\gamma(g)$ in the following way

$$
\begin{aligned}
& 0=\dot{m}_{b}=\dot{Z}_{m} m+Z_{m} \dot{m}=Z_{m} m\left(\left(\ln Z_{m}\right)^{\circ}+(\ln m)^{\circ}\right) \\
& \Rightarrow \gamma(a)=-\frac{d \ln Z_{m}}{d t} \\
& =-\frac{d \ln Z_{m}}{d a} \frac{d a}{d t}=-\frac{d \ln Z_{m}}{d a}(-\varepsilon a+\beta(a))=a \frac{d Z_{m 1}}{d a},
\end{aligned}
$$

where RD equation in $D$-dimension is

$$
\dot{a}=-\varepsilon a+\beta(a)=\beta_{1} a+\beta_{2} a^{2}+\ldots
$$


and $Z_{m 1}$ is the coefficient of the first pole in the $\varepsilon$-expantion of the $Z_{m}$ in $M S$-scheme

$$
Z_{m}(\varepsilon, g)=1+Z_{m 1}(g) \varepsilon^{-1}+Z_{m 2}(g) \varepsilon^{-2}+\ldots
$$

Since $Z_{m}$ does not depend explicitly on $\mu$ and $m$, the $\gamma_{m}$-function is the same in all MS-like schemes.

\subsection{Reparametrization and general method of solution of the RD equation}

$\mathrm{RD}$ equation,

$$
\dot{a}=\beta_{1} a+\beta_{2} a^{2}+\ldots
$$

can be reparametrized,

$$
\begin{aligned}
& a(t)=f(A(t))=A+f_{2} A^{2}+\ldots+f_{n} A^{n}+\ldots=\sum_{n \geq 1} f_{n} A^{n}, \\
& \dot{A}=b_{1} A+b_{2} A^{2}+\ldots=\sum_{n \geq 1} b_{n} A^{n}, \\
& \dot{a}=\dot{A} f^{\prime}(A)=\left(b_{1} A+b_{2} A^{2}+\ldots\right)\left(1+2 f_{2} A+\ldots+n f_{n} A^{n-1}+\ldots\right) \\
& =\beta_{1}\left(A+f_{2} A^{2}+\ldots+f_{n} A^{n}+\ldots\right)+\beta_{2}\left(A^{2}+2 f_{2} A^{3}+\ldots\right)+\ldots \\
& +\beta_{n}\left(A^{n}+n f_{2} A^{n+1}+\ldots\right)+\ldots \\
& =\beta_{1} A+\left(\beta_{2}+\beta_{1} f_{2}\right) A^{2}+\left(\beta_{3}+2 \beta_{2} f_{2}+\beta_{1} f_{3}\right) A^{3}+ \\
& \ldots+\left(\beta_{n}+(n-1) \beta_{n-1} f_{2}+\ldots+\beta_{1} f_{n}\right) A^{n}+\ldots \\
& =\sum_{n, n_{1}, n_{2} \geq 1} A^{n} b_{n_{1}} n_{2} f_{n_{2}} \delta_{n, n_{1}+n_{2}-1} \\
& =\sum_{n, m \geq 1 ; m_{1}, \ldots, m_{k} \geq 0} A^{n} \beta_{m} f_{1}^{m_{1}} \ldots f_{k}^{m_{k}} f\left(n, m, m_{1}, \ldots, m_{k}\right), \\
& f\left(n, m, m_{1}, \ldots, m_{k}\right)=\frac{m !}{m_{1} ! \ldots m_{k} !} \delta_{n, m_{1}+2 m_{2}+\ldots+k m_{k}} \delta_{m, m_{1}+m_{2}+\ldots+m_{k},}, \\
& \quad \\
& b_{1}=\beta_{1}, b_{2}=\beta_{2}+f_{2} \beta_{1}-2 f_{2} b_{1}=\beta_{2}-f_{2} \beta_{1}, \\
& b_{3}=\beta_{3}+2 f_{2} \beta_{2}+f_{3} \beta_{1}-2 f_{2} b_{2}-3 f_{3} b_{1}=\beta_{3}+2\left(f_{2}^{2}-f_{3}\right) \beta_{1}, \\
& b_{4}=\beta_{4}+3 f_{2} \beta_{3}+f_{2}^{2} \beta_{2}+2 f_{3} \beta_{2}-3 f_{4} b_{1}-3 f_{3} b_{2}-2 f_{2} b_{3}, \ldots \\
& b_{n}=\beta_{n}+\ldots+\beta_{1} f_{n}-2 f_{2} b_{n-1}-\ldots-n f_{n} b_{1}, \ldots
\end{aligned}
$$

so, by reparametrization, beyond the critical dimension $\left(\beta_{1} \neq 0\right)$ we can change any coefficient but $\beta_{1}$.

We can fix any higher coefficient with zero value, if we take

$$
f_{2}=\frac{\beta_{2}}{\beta_{1}}, f_{3}=\frac{\beta_{3}}{2 \beta_{1}}+f_{2}^{2}, \ldots, f_{n}=\frac{\beta_{n}+\ldots}{(n-1) \beta_{1}}, \ldots
$$

In this case we have exact classical dynamics in the (external) space-time and simple scale dynamics,

$$
\begin{aligned}
& g=\left(\mu / \mu_{0}\right)^{-\varepsilon} g_{0}=e^{-\varepsilon \tau} g_{0} \\
& \varphi(\tau, t, x)=e^{-(D-2) / 2 \tau} \varphi_{0}(t, x), \\
& \psi(\tau, t, x)=e^{-(D-1) / 2 \tau} \psi_{0}(t, x)
\end{aligned}
$$


We will consider in applications the case when only one of higher coefficient is nonzero.

In the critical dimension of space-time, $\beta_{1}=0$, and we can change by reparametrization any coefficient but $\beta_{2}$ and $\beta_{3}$.

From the relations (5.72), in the critical dimenshion $\left(\beta_{1}=0\right)$, we find that, we can define the minimal form of the RD equation

$$
\dot{A}=\beta_{2} A^{2}+\beta_{3} A^{3}
$$

e.g. $b_{4}=0$ when

$$
f_{3}=\frac{\beta_{4}}{\beta_{2}}+\frac{\beta_{3}}{\beta_{2}} f_{2}+f_{2}^{2}
$$

$f_{2}$ remains arbitrary and we can make choice $f_{2}=0$. We can solve (5.75) as implicit function,

$$
u^{\beta_{3} / \beta_{2}} e^{-u}=c e^{\beta_{2} t}, u=\frac{1}{A}+\frac{\beta_{3}}{\beta_{2}}
$$

than, as in the noncritical case, explicit solution will be given by reparametrization representation.

If we know somehow the coefficients $\beta_{n}$, e.g. for first several exact and for others asymptotic values (see e.g. [Kazakov, Shirkov, 1980]) than we can construct reparametrization function (5.70) and find the dynamics of the running coupling constant. This is similar to the action-angular canonical transformation of the analytic mechanics (see e.g. [Faddeev, Takhtajan, 1987]).

Statement: The series for $a$ is p-adically convergent, when $\beta_{n}$ and $A$ are rational numbers.

\subsection{Reparametrization of the anomalous dimensions}

Let us take the the anomalous dimension of some quantity (see e.g. [Smirnov, CALC 2012], [Moch, CALC 2012])

$$
\gamma(a)=\gamma_{1} a+\gamma_{2} a^{2}+\gamma_{3} a^{3}+\ldots
$$

and make reparametrization

$$
\begin{gathered}
a=f(A)=A+f_{2} A^{2}+f_{3} A^{3}+\ldots \\
\gamma(a)=\gamma_{1}\left(A+f_{2} A^{2}+f_{3} A^{3}+\ldots\right)+\gamma_{2}\left(A^{2}+2 f_{2} A^{3}+\ldots\right)+\gamma_{3}\left(A^{3}+\ldots\right)+\ldots \\
=\Gamma_{1} A+\Gamma_{2} A^{2}+\Gamma_{3} A^{3}+\ldots \\
\Gamma_{1}=\gamma_{1}, \Gamma_{2}=\gamma_{2}+\gamma_{1} f_{2}, \Gamma_{3}=\gamma_{3}+2 \gamma_{2} f_{2}+\gamma_{1} f_{3}, \ldots
\end{gathered}
$$

When $\gamma_{1} \neq 0$, we can take $\Gamma_{n}=0, n \geq 2$, if we define $f_{n}$ as

$$
f_{2}=-\frac{\gamma_{2}}{\gamma_{1}}, f_{3}=-\frac{\gamma_{3}+2 \gamma_{2} f_{2}}{\gamma_{1}}=-\frac{\gamma_{3}-2 \gamma_{2}^{2} / \gamma_{1}}{\gamma_{1}}, \ldots
$$

So, we get the exact value for the anomalous dimension

$$
\gamma(A)=\gamma_{1} A=\gamma_{1} f^{-1}(a)=\gamma_{1}\left(a+\gamma_{2} / \gamma_{1} a^{2}+\gamma_{3} / \gamma_{1} a^{3}+\ldots\right)
$$




\subsection{Renormdynamic functions (RDF)}

We will call RDF functions $g_{n}=f_{n}(t)$, which are solutions of the RD motion equations

$$
\dot{g}_{n}=\beta_{n}(g), 1 \leq n \leq N .
$$

In the simplest case of one coupling constant, the function $g=f(t)$, is constant $g=g_{c}$ when $\beta\left(g_{c}\right)=0$, or is invertible (monotone). Indeed,

$$
\dot{g}=f^{\prime}(t)=f^{\prime}\left(f^{-1}(g)\right)=\beta(g) .
$$

Each monotone interval ends by UV and IR fixed points and describes corresponding phase of the system.

\subsection{Nonperturbative renormdynamic functions}

Based on real experiments and computer simulations, quantum gauge theory in four dimensions is believed to have a mass gap. This is one of the most fundamental facts that makes the Universe the way it is.

In the lattice (gauge) theory approach to the renormdynamics (see, e.g. [Makhaldiani, 1986]), coupling constant dynamics were calculated for $S U$ (3) Yang-Mills model [Bogolubsky et al, 2009]. The result is in agreement with perturbative calculations at small scales; at an intermediate scale the coupling constant reaches its maximum $(\simeq 1$.); than decrease. So, at the maximum, we may have nontrivial zero of the $\beta$-function, which corresponds to the conformal invariance of the gluodynamics at this point. Beyond this point we have another phase, strong coupling phase with decreasing coupling constant similar (identical?!) to the abelian (monopole?) theory.

Note that, in the case of the two coupling constants,

$$
\begin{aligned}
& \dot{g}_{1}=\beta_{1}\left(g_{1}, g_{2}\right), \\
& \dot{g}_{2}=\beta_{2}\left(g_{1}, g_{2}\right),
\end{aligned}
$$

we can reformulate $\mathrm{RD}$ as

$$
\begin{aligned}
& g_{1} \equiv g ; g_{2}=f_{2}(t) \equiv \tau, \\
& \frac{d g_{1}}{d g_{2}}=\frac{d g}{d \tau} \equiv \dot{g}=\beta(g, \tau)=\frac{\beta_{1}(g, \tau)}{\beta_{2}(g, \tau)}
\end{aligned}
$$

and RDF must fulfil corresponding restrictions. E.g. if

$$
g_{1}=f_{1}(t)=g=f(\tau)=f\left(f_{2}(t)\right), g_{2}=f_{2}(t)=\tau
$$

So, if we approximate the form of the curve near maximum as

$$
a(t)=a_{c}-b\left|t-t_{c}\right|^{n}
$$

for the $\beta$-function we obtain

$$
\dot{a}=\beta(a, t)=\operatorname{sign}\left(t_{c}-t\right) b n\left(\frac{a_{c}-a}{b}\right)^{\frac{n-1}{n}} .
$$

Of course this is not usual $\beta$-function, function of $a$ only. It depends also on $t$. For $t>t_{c}$ we have perturbative phase. For $n>1, \beta\left(a_{c}, t\right)=0$. Explicit dependence on time variable in one coupling case indicates on implicit two coupling case. 


\subsection{QCD, parton model, valence quarks and $\alpha_{s}=2$}

While it has been well established in the perturbative regime at high energies, QCD still lacks a comprehensive solution at low and intermediate energies, even 40 years after its invention. In order to deal with the wealth of non-perturbative phenomena, various approaches are followed with limited validity and applicability. This is especially also true for lattice QCD, various functional methods, or chiral perturbation theory, to name only a few. In neither one of these approaches the full dynamical content of QCD can yet be included. Basically, the difficulties are associated with a relativistically covariant treatment of confinement and the spontaneous breaking of chiral symmetry, the latter being a well-established property of QCD at low and intermediate energies. As a result, most hadron reactions, like resonance excitations, strong and electroweak decays etc., are nowadays only amenable to models of QCD. Most famous is the constituent-quark model (CQM), which essentially relies on a limited number of effective degrees of freedom with the aim of encoding the essential features of low- and intermediate-energy QCD.

The CQM has a long history, and it has made important contributions to the understanding of many hadron properties, think only of the fact that the systematization of hadrons in the standard particle-data base follows the valence-quark picture.

It was noted [Voloshin, Ter-Martyrosian, 1984] that parton densities given by the following solution

$$
\begin{aligned}
& M_{2}\left(Q^{2}\right)=\frac{3}{25}+\frac{2}{3} \omega^{-32 / 81}+\frac{16}{75} \omega^{-50 / 81} \\
& \bar{M}_{2}\left(Q^{2}\right)=M_{2}^{s}\left(Q^{2}\right)=\frac{3}{25}-\frac{1}{3} \omega^{-32 / 81}+\frac{16}{75} \omega^{-50 / 81} \\
& M_{2}^{G}\left(Q^{2}\right)=\frac{16}{25}\left(1-\omega^{-50 / 81}\right), \\
& \omega=\frac{\alpha_{s}\left(m^{2}\right)}{\alpha_{s}\left(Q^{2}\right)}, Q^{2} \in(5,20) G e V^{2}, b=9, \alpha_{s}\left(Q^{2}\right) \simeq 0.2
\end{aligned}
$$

of the Altarelli-Parisi equation

$$
\begin{aligned}
& \dot{M}=A M \\
& M^{T}=\left(M_{2}, \bar{M}_{2}, M_{2}^{s}, M_{2}^{G}\right), \\
& M_{2}=\int_{0}^{1} d x x(u(x)+d(x)), \bar{M}_{2}=\int_{0}^{1} d x x(\bar{u}(x)+\bar{d}(x)), \\
& M_{2}^{s}=\int_{0}^{1} d x x(s(x)+\bar{s}(x)), M_{2}^{G}=\int_{0}^{1} d x x G(x), \\
& A=\left(\begin{array}{cccc}
32 / 9 & 0 & 0 & -2 / 3 \\
0 & 32 / 9 & 0 & -2 / 3 \\
0 & 0 & 32 / 9 & -2 / 3 \\
-32 / 9 & -32 / 9 & -32 / 9 & 2
\end{array}\right)
\end{aligned}
$$

with the following "valence quark" initial condition at a scale $m$

$$
\begin{aligned}
& M_{2}\left(m^{2}\right)=1, \bar{M}_{2}\left(m^{2}\right)=M_{2}^{s}\left(m^{2}\right)=M_{2}^{G}\left(m^{2}\right)=0, \\
& \alpha_{s}\left(m^{2}\right)=2,
\end{aligned}
$$

gives the experimental values

$$
M_{2}=0.44, \bar{M}_{2}=M_{2}^{s}=0.04, M_{2}^{G}=0.48
$$


So, for valence quark VQCD, $\alpha_{s}\left(m^{2}\right)=2$. We have seen, that for $\pi \rho N$ model $\alpha_{\pi \rho N}=3$, and for $\pi N$ model $\alpha_{\pi N}=13$. It is nice that $\alpha_{s}^{2}+\alpha_{\pi \rho N}^{2}=\alpha_{\pi N}$. This relation can be seen, e.g., by considering pion propagator in the low energy $\pi N$ model and in superposition of higher energy VQCD and $\pi \rho N$ models.

\subsection{Quarkonia, potential and space dimension renormdynamics}

Phenomenological approach to the nonrelativistic potential-model study of $\Upsilon$ and $\psi$ spectra leads to a static Coulombic Power-law potential of the form

$$
\begin{aligned}
V(r)=a(r) r^{2-d(r)} \sim 1 / r, r & \sim 0.1 f m \\
r, r & \sim 1 . f m
\end{aligned}
$$

E.g. in the case of the $\Upsilon$ and small $r$

$$
V(r)=\frac{4}{3} \frac{\alpha_{s}}{r}, \alpha_{s}=\frac{2 \pi}{b \ln r \Lambda}, b=9 .
$$

This behavior corresponds not only to the running fine structure constant but also to the running space dimension. Confinement-the point-like hadrons on the scales higher than hadronic, corresponds to the zero dimensional space for hadron constituents.

RD equations of QCD beyond the critical dimention has explicit dependence on the space dimension. When the dimension becomes running we should consider two dimensional renormdinamics

$$
\begin{aligned}
& \dot{a_{1}}=\beta_{1}\left(a_{1}, a_{2}\right), a_{1}=a \\
& \dot{a_{2}}=\beta_{2}\left(a_{1}, a_{2}\right), a_{2}=d
\end{aligned}
$$

\subsection{Low energy unification of the two abelian couplings}

At the critical point we may have low energy unification of the two abelian couplings, weakelectromagnetic and strong-monopole couplings. According to the Dirac quantization rule, for the electron- $e$ and monopole- $g$ charges we have

$$
e g=2 \pi n, n= \pm 1, \pm 2, \ldots
$$

so, at the selfdual, critical, point, we have prediction:

$$
\alpha_{e}=\alpha_{g}=\frac{n}{2}, n=1,2, \ldots
$$

The minimal-fundamental value of the unified coupling constant is $\alpha=1 / 2$. In the Schwinger monopol case, only even $n$ is permitted and the minimal value at the unification point is 1 . The next value is 2 . These two values of coupling constant are connected as UV and IR fixed points of one monotone RD interval. 


\subsection{Stability of the states of dynamical systems}

If we have a solution $x_{n}=x_{0 n}$ (a state) of the following system of motion equations (of the corresponding dynamical system)

$$
\dot{x}_{n}=f_{n}(x), 1 \leq n \leq N,
$$

we can consider the question of stability of the solution, the existence of the solutions of the type $x_{n}=x_{0 n}+g_{n}$, for small values of $g_{n}$. If there are solutions with rising $g_{n}$, of the corresponding motion equations

$$
\begin{aligned}
& \dot{g}_{n}=\beta_{n}(g), \beta_{n}(g)=f_{n}\left(x_{0}+g\right)-f_{n}\left(x_{0}\right) \\
& =\beta_{1 n m} g_{m}+\beta_{2 n m k} g_{m} g_{k}+\ldots, \beta_{k n \ldots m}=f^{(n \ldots m)}\left(x_{0}\right)
\end{aligned}
$$

we say that the solution $x_{0 n}$ is not stable.

The linear approximation, we transform into diagonal form,

$$
\begin{aligned}
& \dot{g}_{n}=\beta_{1 n m} g_{m}, h_{n}=A_{n m} g_{m}, \\
& \dot{h}_{n}=\lambda_{n} h_{n}, \lambda_{n} \delta_{n m}=\left(A \beta_{1} A^{-1}\right)_{n m},
\end{aligned}
$$

if all of the $\lambda_{n}$ are purely imaginary $\lambda_{n}=i \omega_{n}$, we have stable solution (in the linear approximation): small deviations remain small. If real parts of all $\lambda_{n}$ are negative, we have asymptotic stability: deviations decrease. If some $\lambda_{n}$ are zero, we have undefined case. In regular case, when the matrix $\beta_{1}$ has inverse, by reparametrization trick we can construct the formal solution of the nonlinear equation for $g_{n}$, and try to investigate its convergence properties.

\subsection{Nambu - Poisson formulation of Renormdynamics}

In the case of several integrals of motion, $H_{n}, 1 \leq n \leq N$, we can formulate Renormdynamics as Nambu - Poisson dynamics (see e.g. [Makhaldiani, 2007])

$$
\dot{\varphi}(x)=\left[\varphi(x), H_{1}, H_{2}, \ldots, H_{N}\right],
$$

where $\varphi$ is an observable as a function of the coupling constants $x_{m}, 1 \leq m \leq M$.

In the case of Standard model [Weinberg, 1995], we have three coupling constants, $M=3$.

\subsection{Hamiltonian extension of the Renormdynamics}

The renormdynamic motion equations

$$
\dot{g}_{n}=\beta_{n}(g), 1 \leq n \leq N
$$

can be presented as nonlinear part of a Hamiltonian system with linear part

$$
\dot{\Psi}_{n}=-\frac{\partial \beta_{m}}{\partial g_{n}} \Psi_{m},
$$

Hamiltonian and canonical Poisson bracket as

$$
H=\sum_{n=1}^{N} \beta(g)_{n} \Psi_{n},\left\{g_{n}, \Psi_{m}\right\}=\delta_{n m}
$$


In this extended version, we can define optimal control theory approach [Pontryagin, 1983] to the unified field theories. We can start from the unified value of the coupling constant, e.g. $\alpha^{-1}(M)=29.0 \ldots$ at the scale of unification $M$, put the aim to reach the SM scale with values of the coupling constants measured in experiments, and find optimal threshold corrections to the RD coefficients [Makhaldiani, 2010].

\subsection{Renormdynamic equation for effective action}

For connected vertex functions $\Gamma_{n},(\mathbb{L} \cdot \mathrm{W})$

$$
\begin{aligned}
& \Gamma_{n}\left(x_{1}, x_{2}, \ldots, x_{n} ; g, m, \mu\right)=Z^{n / 2}(\mu) \Gamma_{0 n}\left(x_{1}, x_{2}, \ldots, x_{n} ; g_{0}, m_{0}\right), \\
& \left(D-\frac{n}{2} \gamma\right) \Gamma_{n}(x ; g, m, \mu)=0
\end{aligned}
$$

For effective action $S_{q}$,

$$
\begin{aligned}
& \left(D-\frac{1}{2} \gamma \int d x \phi(x) \frac{\delta}{\delta \phi(x)}\right) S_{q}(\phi)=0 \\
& \left(D-\frac{1}{2} \gamma \phi \frac{\partial}{\partial \phi}\right) V(\phi)=0, V(\phi)=\left.S_{q}(\phi(x))\right|_{\phi(x)=\phi=c o n s t},
\end{aligned}
$$

where $V(\phi)$ is effective potential.

For the effective potential in the RD (conformal) fixed point, $\gamma(g)=\gamma\left(g_{c}\right) \equiv \gamma_{c}$ we have the following wave equation and corresponding (auto model) solution

$$
\begin{aligned}
& \left(\partial_{t}-\frac{\gamma_{c}}{2} \partial_{z}\right) V=0, \\
& V(\phi, \mu)=f(z+v t)=F\left(\frac{\phi}{\mu^{v}}\right), t=\ln \frac{\mu}{\mu_{0}}, z=\ln \frac{\phi}{\phi_{0}}, v=\frac{\gamma_{c}}{2} .
\end{aligned}
$$

\subsection{Finite temperature and density QCD}

The fundamental quark and gluon degrees of freedom are the relevant ones at high temperatures and/or densities. Since these degrees of freedom are confined in the low temperature and density regime there must be a quark and/or gluon (de)confinement phase transition.

It is difficult to describe the phase transition because there is not known a local parameter which can be linked to confinement. We consider the fractal dimension of the hadronic/quarkgluon space as order parameter of (de)confinement phase transition. It has value less than 3 in the abelian, hadronic, phase, and more than 3 , in nonabelian, quark-gluon, phase.

\subsection{Ultraviolet (Infrared) fixed point for QCD (QED)}

Perturbation theory results for QCD (QED) give negative (positive) $\beta$-function, in one loop approximation

$$
\begin{aligned}
& \dot{a}=\beta_{2} a^{2}, a=\left(\frac{g}{4 \pi}\right)^{2} \\
& Q C D: \beta_{2}=\left(\frac{2 n_{f}}{3}-11\right), \\
& Q E D: \beta_{2}=\frac{4}{3}
\end{aligned}
$$


So, running coupling constant vanishes at higher (low) energy. For QCD this property named as asymptotic freedom gives the scaling behavior of observable quantities in good agreement with experimental data. Small value of the coupling constant may describe small deviation from the scaling. Infrared zero value of the QED coupling constant contradicts with experiments. Small value of the coupling constant equal to the observable value of the fine structure constant $\alpha^{-1}=$ 137.036, in the infrared (low energy) limit, will be good solution of the zero-charge problem. For this, we will consider the QCD (QED) beyond the critical dimension of the space-time.

Corresponding $\beta$-function

$$
\beta(a, \varepsilon)=-\varepsilon a+\beta(a),
$$

has stable ultraviolet (infrared) fixed point for negative (positive) value of $\varepsilon$,

$$
\varepsilon=\beta(a) / a=\beta_{2} a+\ldots
$$

We have predictions for deviation from the classical value of the space dimension $3, d=3-2 \varepsilon$, in one loop approximation

$$
\begin{aligned}
& \varepsilon_{Q E D}=\frac{4}{3} \frac{\alpha}{4 \pi}=\frac{\alpha}{3 \pi}=7.8 \times 10^{-4}, \\
& \varepsilon_{Q C D}=-7 \frac{\alpha_{s}}{4 \pi}=-0.06, n_{f}=6, \alpha_{s}=0.1
\end{aligned}
$$

\subsection{Two TeV scale unification of the standard model coupling constants}

According to the LEP and Tevatron data, the standard model coupling constants at the Z-boson mass scale take the values (see, e.g. [Kazakov, 2004])

$$
\begin{aligned}
& \alpha_{1}\left(m_{Z}\right)=0.017, \alpha_{1}\left(m_{Z}\right)^{-1}=58.8 \\
& \alpha_{2}\left(m_{Z}\right)=0.034, \alpha_{2}\left(m_{Z}\right)^{-1}=29.4 \\
& \alpha_{3}\left(m_{Z}\right)=0.118, \alpha_{3}\left(m_{Z}\right)^{-1}=8.47
\end{aligned}
$$

Our aim is to consider RD equation in critical dimension for weak interaction part of the $\mathrm{SM}\left(\varepsilon_{2}=0\right)$; RD equations for the electromagnetic and strong interaction parts beyond critical dimension $\left(\varepsilon_{1}, \varepsilon_{3} \neq 0\right)$; reach unification (equality) of the three couplings at the $\mathrm{TeV}$ scale in the point $\alpha_{u}^{-1}=31.0$

The solution of the one loop RD equation beyond critical dimension

$$
\begin{aligned}
& \dot{a}=-\varepsilon a+k a^{2}, \\
& a=\frac{\alpha}{4 \pi}=\left(\frac{g}{4 \pi}\right)^{2}, t=\ln \frac{Q^{2}}{m_{Z}^{2}},
\end{aligned}
$$

is

$$
\begin{aligned}
& a_{n}(t)^{-1}=\frac{k_{n}}{\varepsilon}+c_{n} e^{\varepsilon_{n} t}, n=1,3 \\
& c_{n}=a_{n}\left(m_{Z}\right)^{-1}-\frac{k_{n}}{\varepsilon_{n}}, \\
& k_{n}=\left(\frac{41}{10},-7\right) .
\end{aligned}
$$


The solution of the RD equation in critical dimension

$$
\dot{a}_{2}=k_{2} a_{2}^{2}, k_{2}=-\frac{19}{6}
$$

is

$$
a_{2}^{-1}(t)=a_{2}^{-1}\left(m_{Z}\right)+k_{2} t
$$

From the last expression, having unification value, $\alpha_{2}^{-1}\left(t_{u}\right)=\alpha_{u}^{-1}=31.0$ we define the unification scale

$$
\begin{aligned}
& t_{u}=\left(a_{2}^{-1}\left(t_{u}\right)-a_{2}^{-1}\left(m_{Z}\right)\right) / k_{2} \\
& =4 \pi \times 1.6 \times \frac{6}{19}=6.35, \\
& Q_{u}=23.9 m_{Z}=2182 \mathrm{GeV}, \\
& m_{Z}=91.2 \mathrm{GeV}
\end{aligned}
$$

Solution of the RD equation beyond the critical dimension for electrodynamic constant,

$$
\dot{a}=-\varepsilon a+b a^{2}, b=\frac{41}{10},
$$

is

$$
a^{-1}(t)=\frac{b}{\varepsilon}+\left(a^{-1}\left(m_{Z}\right)-\frac{b}{\varepsilon}\right) e^{\varepsilon t}
$$

The condition of the unification

$$
\left(b \varepsilon^{-1}-a^{-1}\left(t_{u}\right)\right)=\left(b \varepsilon^{-1}-a^{-1}\left(m_{Z}\right)\right) e^{\varepsilon t_{u}}
$$

defines the value $\varepsilon_{1}=-0.093$ Unification takes place in dimension $d=4-2 \varepsilon_{1}=4.186$

For the strong coupling constant beyond the critical dimension,

$$
\dot{a}=-\varepsilon a-b a^{2}, b=7,
$$

the solution is

$$
a^{-1}(t)=-\frac{b}{\varepsilon}+\left(\frac{b}{\varepsilon}+a^{-1}\left(m_{Z}\right)\right) e^{t \varepsilon},
$$

the unification condition

$$
\left(b \varepsilon^{-1}+a^{-1}\left(t_{u}\right)\right)=\left(b \varepsilon^{-1}+a^{-1}\left(m_{Z}\right)\right) e^{\varepsilon t_{u}}
$$

defines $\varepsilon=0.168$ Unification takes place in the dimension $d=4-2 \varepsilon=3.66$

Let us consider unification at the point $\alpha^{-1}\left(t_{u}\right)=29.0$, the low energy unification,

$$
\begin{aligned}
& t_{u l}=\left(\alpha_{2}^{-1}\left(t_{u l}\right)-a_{2}^{-1}\left(m_{Z}\right)\right) / k_{2} \\
& =-4 \pi \times 0.4 \times \frac{6}{19}=-1.59, \\
& Q_{u l}=0.45 m_{Z}=41.2 \mathrm{GeV}
\end{aligned}
$$


For electrodynamic case unification condition

$$
\frac{41}{10}-4 \pi 29 \varepsilon=\left(\frac{41}{10}-4 \pi 58.8 \varepsilon\right) e^{-1.59 \varepsilon},
$$

gives the values $\varepsilon_{1}=0.453, d_{e l}=3.09=2.09+1$ dimensional space-time.

For strong coupling constant unification condition

$$
7+4 \pi \varepsilon \times 29=(7+4 \pi \varepsilon \times 8.47) e^{-1.59 \varepsilon}
$$

gives $\varepsilon_{3}=-0.8121, d_{s l}=5.624$

5.26 At what scale $\alpha^{-1}=137$ ?

The low energy value of the QED $\alpha^{-1}=137.036 \ldots$

Let us find the scale at which $\alpha^{-1}=137$ if

$$
\begin{aligned}
& \alpha^{-1}\left(m_{Z}\right)=\frac{5}{3 \cos ^{2} \theta_{W}} \alpha_{1}^{-1}\left(m_{Z}\right)=128.978 \pm 0.027 \simeq 129 \\
& \sin ^{2} \theta_{W}=0.23146 \pm 0.00017 \simeq 0.2315 \\
& \alpha_{1}^{-1}\left(m_{Z}\right)=58.8 \\
& \alpha^{-1}\left(m_{Z}\right)=\frac{5}{3 \times 0.7685} \times 58.8=127.52 \simeq 128
\end{aligned}
$$

Now take one loop RD evolution to the 137 ,

$$
\begin{aligned}
& t_{l}=\left(a_{1}^{-1}\left(t_{l}\right)-a_{1}^{-1}\left(m_{Z}\right)\right) / k_{1} \\
& =-4 \pi \times 8 . \times \frac{10}{41}=-24.5, \\
& Q_{l} \simeq 5 \times 10^{-6} m_{Z} \simeq 5 \times 10^{-4} m_{p} \simeq m_{e}
\end{aligned}
$$

\section{Renormdynamics of observable quantities in high energy physics}

Let us consider $l$-particle semi-inclusive distribution

$$
\begin{aligned}
& F_{l}(n, q)=\frac{d^{l} \sigma_{n}}{\bar{d} q_{1} \ldots \bar{d} q_{l}}=\frac{1}{n !} \int \prod_{i=1}^{n} \bar{d} q_{i}^{\prime} \delta\left(p_{1}+p_{2}-\Sigma_{i=1}^{l} q_{i}-\sum_{i=1}^{n} q_{i}^{\prime}\right) \\
& \left.\cdot \mid M_{n+l+2}\left(p_{1}, p_{2}, q_{1}, \ldots, q_{l}, q_{1}^{\prime}, \ldots, q_{n}^{\prime} ; g(\mu), m(\mu)\right), \mu\right)\left.\right|^{2}, \\
& \bar{d} p \equiv \frac{d^{3} p}{E(p)}, E(p)=\sqrt{p^{2}+m^{2}} .
\end{aligned}
$$

From the renormdynamic equation

$$
D M_{n+l+2}=\frac{\gamma}{2}(n+l+2) M_{n+l+2},
$$

we obtain

$$
\begin{aligned}
& D F_{l}(n, q)=\gamma(n+l+2) F_{l}(n, q), \\
& D F_{l}(q)=\gamma(<n>+l+2) F_{l}(q), \\
& D<n^{k}(q)>=\gamma\left(<n^{k+1}(q)>-<n^{k}(q)><n(q)>\right), \\
& D C_{k}=\gamma<n(q)>\left(C_{k+1}-C_{k}\left(1+k\left(C_{2}-1\right)\right)\right) \\
& F_{l}(q) \equiv \frac{d^{l} \sigma}{\bar{d} q_{1} \ldots \bar{d} q_{l}}=\sum_{n} \frac{d^{l} \sigma_{n}}{\bar{d} q_{1} \ldots \bar{d} q_{l}},<n^{k}(q)>=\frac{\sum_{n} n^{k} d^{l} \sigma_{n} / \bar{d} q^{l}}{\sum_{n} d^{l} \sigma_{n} / \bar{d} q^{l}} \\
& C_{k}=\frac{<n^{k}(q)>}{<n(q)>^{k}}
\end{aligned}
$$




\subsection{Scaling relations for multi particle cross sections}

From dimensional considerations, the following combination of cross sections [Koba et al, 1972] must be universal function

$$
<n>\frac{\sigma_{n}}{\sigma}=\Psi\left(\frac{n}{<n>}\right) .
$$

Corresponding relation for the inclusive cross sections is [Matveev et al, 1976].

$$
<n(p)>\frac{d \sigma_{n}}{\bar{d} p} / \frac{d \sigma}{\bar{d} p}=\Psi\left(\frac{n}{<n(p)>}\right) .
$$

Indeed, let us define $n$-dimension of observables [Makhaldiani, 1980]

$$
[n]=1,\left[\sigma_{n}\right]=-1, \sigma=\Sigma_{n} \sigma_{n},[\sigma]=0,[<n>]=1 .
$$

The following expression does not depend on any dimensional quantities and must have a corresponding universal form

$$
P_{n}=<n>\frac{\sigma_{n}}{\sigma}=\Psi\left(\frac{n}{<n>}\right) .
$$

Let us find an explicit form of the universal functions using renormdynamic equations.

From the definition of the moments we have

$$
C_{k}=\int_{0}^{\infty} d x x^{k} \Psi(x)
$$

so they are universal parameters,

$$
\begin{aligned}
& D C_{k}=0 \Rightarrow C_{k+1}=\left(1+k\left(C_{2}-1\right)\right) C_{k} \Rightarrow \\
& C_{k}=\left(1+(k-1)\left(C_{2}-1\right)\right) \ldots\left(1+2\left(C_{2}-1\right)\right) C_{2} .
\end{aligned}
$$

Now we can invert momentum transform and find (see [Makhaldiani, 1980] ) universal functions [Ernst, Schmit, 1976], [Darbaidze et al, 1978].

$$
\begin{aligned}
& \Psi(z)=\frac{1}{2 \pi i} \int_{-i \infty}^{+i \infty} d n z^{-n-1} C_{n}=\frac{c^{c}}{\Gamma(c)} z^{c-1} e^{-c z}, \\
& C_{2}=1+\frac{1}{c}
\end{aligned}
$$

The value of the parameter $c$ can be measured from the dispersion low,

$$
\begin{aligned}
& D=\sqrt{\left\langle n^{2}>-<n\right\rangle^{2}}=\sqrt{C_{2}-1}<n>=A<n>, \\
& A=\frac{1}{\sqrt{c}} \simeq 0.6, c=2.8 ; \\
& (c=3, A=0.58)
\end{aligned}
$$

which is in accordance with $n$-dimension counting. 


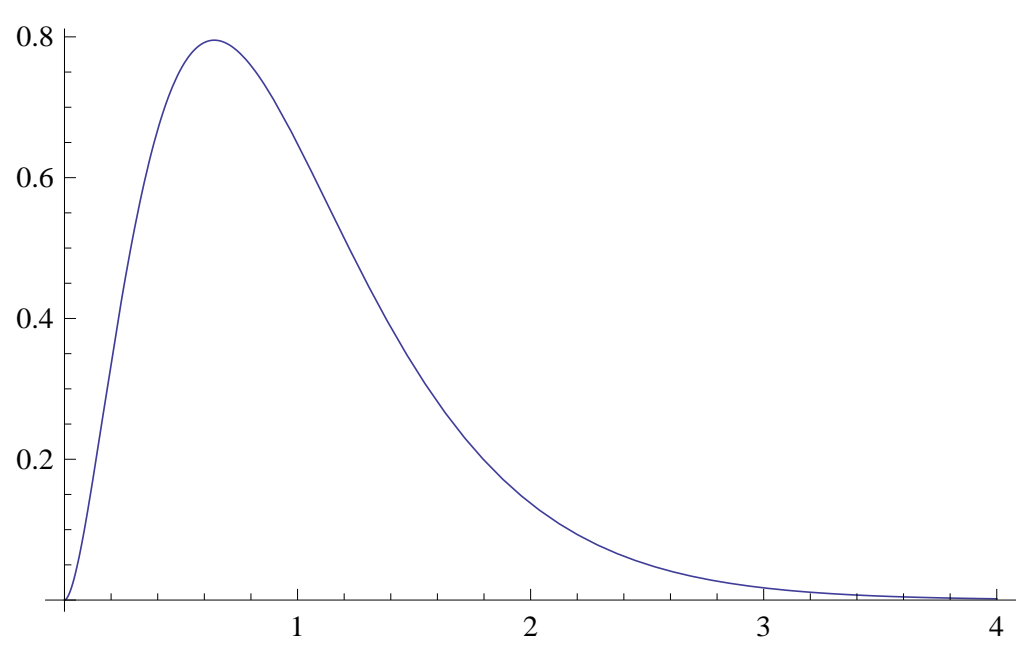

Figure 1: KNO distribution, $\Psi(z)$, with $c=2.8$

\section{2 $1 /<n>$ correction to the scaling function}

We can calculate also $1 /<n>$ correction to the scaling function

$$
\begin{aligned}
& <n>\frac{\sigma_{n}}{\sigma}=\Psi=\Psi_{0}\left(\frac{n}{\langle n\rangle}\right)+\frac{1}{\langle n\rangle} \Psi_{1}\left(\frac{n}{\langle n\rangle}\right), \\
& C_{k}=C_{k}^{0}+\frac{1}{\langle n\rangle} C_{k}^{1}, \\
& C_{k}^{0}=\int_{0}^{\infty} d x x^{k} \Psi_{0}(x), C_{k}^{1}=\int_{0}^{\infty} d x x^{k} \Psi_{1}(x), \\
& \Psi_{1}(z)=\frac{1}{2 \pi i} \int_{-i \infty}^{+i \infty} d n z^{-n-1} C_{n}^{1}=\frac{C_{2}^{1} c^{2}}{2}\left(z-2+\frac{c-1}{c z}\right) \Psi_{0}
\end{aligned}
$$

\subsection{Characteristic function for KNO}

The characteristic function we define as

$$
\Phi(t)=\int_{0}^{\infty} d x e^{t x} \Psi(x)=(1-t / c)^{-c}, \operatorname{Re}(t)<c
$$

For the moments of the distribution, we have

$$
\Phi^{(k)}(0)=C_{k}=(-c)(-c-1) \ldots(-c-k+1)(-1 / c)^{k}=\frac{\Gamma(c+k)}{\Gamma(c) c^{k}}
$$

Note that it is an infinitely divisible characteristic function, i.e.

$$
\Phi(t)=\left(\Phi_{n}(t)\right)^{n}, \Phi_{n}(t)=(1-t / c)^{-c / n}
$$

If we calculate observable(mean) value of $x$, we find

$$
\begin{aligned}
& <x>=\Phi^{\prime}(0)=n \Phi(0)_{n}{ }^{\prime}=n<x>_{n}, \\
& <x>_{n}=\frac{<x>}{n}
\end{aligned}
$$

For the second moment and dispersion, we have

$$
<x^{2}>=\Phi^{(2)}(0)=n<x^{2}>_{n}+n(n-1)<x>_{n}^{2},
$$




$$
\begin{aligned}
& \left.\left.\mathrm{D}^{2}=<x^{2}>-<x\right\rangle^{2}=n\left(<x^{2}>_{n}-<x\right\rangle_{n}^{2}\right)=n \mathrm{D}_{n}^{2} \\
& \mathrm{D}_{n}^{2}=\frac{\mathrm{D}^{2}}{n}=\frac{\mathrm{D}^{2}}{\langle x\rangle}<x>_{n}
\end{aligned}
$$

In a sense, any Hamiltonian quantum (and classical) system can be described by infinitely divisible distributions, because in the functional integral formulation, we use the following step

$$
U(t)=e^{-i t H}=\left(e^{-i \frac{t}{N} H}\right)^{N}
$$

In the case of our scalar field theory (2.]),

$$
\begin{aligned}
& L(\varphi)=\frac{1}{2} \partial_{\mu} \varphi \partial^{\mu} \varphi-\frac{m^{2}}{2} \varphi^{2}-\frac{g}{n} \varphi^{n} \\
& =g^{\frac{2}{2-n}}\left(\frac{1}{2} \partial_{\mu} \phi \partial^{\mu} \phi-\frac{m^{2}}{2} \phi^{2}-\frac{1}{n} \phi^{n}\right)
\end{aligned}
$$

so, to the constituent field $\phi_{N}$ corresponds higher value of the coupling constant,

$$
g_{N}=g N^{\frac{n-2}{2}}
$$

For weak nonlinearity, $n=2+2 \varepsilon, d=2 / \varepsilon+2, g_{N}=g\left(1+\varepsilon \ln N+O\left(\varepsilon^{2}\right)\right)$

\subsection{Closed equation of renormdynamics for the generating function of the observables}

Let us consider a generating function of the topological crossections

$$
\begin{aligned}
& F(h, g, m, \mu)=\Sigma_{n \geq 2} h^{n} \sigma_{n}, \\
& \sigma_{n}=\left.\frac{1}{n !} \frac{d^{n}}{d h^{n}} F\right|_{h=0}, \\
& \sigma=\left.F\right|_{h=1}, \quad<n>=\left.\frac{d}{d h} \ln F\right|_{h=1}, \ldots
\end{aligned}
$$

It is natural that for the generating function we have closed renormdynamic equation [Makhaldiani, 1980]

$$
\begin{aligned}
& \left(D-\gamma\left(\frac{h \partial}{\partial h}+2\right)\right) F=0, \\
& F(h(\mu), g(\mu), m(\mu), \mu)=F(h(\bar{\mu}), g(\bar{\mu}), m(\bar{\mu}), \bar{\mu}) \exp \left(2 \int_{\bar{\mu}}^{\mu} \frac{d \rho}{\rho} \gamma(g(\rho))\right), \\
& \bar{h}=\bar{h}(\bar{\mu})=h(\mu) \exp \left(\int_{\mu}^{\bar{\mu}} \frac{d \rho}{\rho} \gamma(g(\rho))\right), \\
& \bar{m}=\bar{m}(\bar{\mu})=m(\mu) \exp \left(\int_{\mu}^{\bar{\mu}} \frac{d \rho}{\rho} \eta(g(\rho))\right), \int_{g}^{\bar{g}} \frac{d g}{\beta(g)}=\ln \frac{\bar{\mu}}{\mu}
\end{aligned}
$$

\subsection{Explicit form of Generating function in the case of KNO scaling}

Let us find generating function in the case of KNO scaling. From the definition of Generating function and using topological cross section from $\mathrm{KNO}$, we find

$$
\begin{aligned}
& F(h)=\sum_{n} h^{n} \frac{\sigma}{<n>} \Psi\left(\frac{n}{<n>}\right)=\frac{\sigma}{<n>} \sum \Psi\left(\frac{n}{<n>}\right) h^{n} \\
& =\frac{\sigma}{<n>} \Psi\left(\frac{\delta}{<n>}\right) \frac{h^{2}}{1-h}, \delta \equiv h \frac{d}{d h}, q^{\delta} f(h)=f(q h),
\end{aligned}
$$


Now we can find more concrete form of the generating function, with the explicit form of KNO function,

$$
\begin{aligned}
& \left(\frac{\delta}{<n>}\right)^{c-1} \exp \left(-c \frac{\delta}{<n>}\right) \frac{h^{2}}{1-h}=\left(\frac{\delta}{<n>}\right)^{c-1} \frac{q^{2} h^{2}}{1-q h} \\
& =\frac{1}{<n>^{c-1}} \frac{1}{\Gamma(1-c)} \int_{0}^{\infty} \frac{d t}{t^{c}} \frac{q^{2} h^{2} e^{-2 t}}{1-q h e^{-t}},
\end{aligned}
$$

so

$$
\begin{aligned}
& F(h)_{K N O}=\frac{c^{c}}{\Gamma(c)} \frac{\sigma}{<n>^{c}} \frac{1}{\Gamma(1-c)} \int_{0}^{\infty} \frac{d t}{t^{c}} \frac{q^{2} h^{2} e^{-2 t}}{1-q h e^{-t}}, \\
& q=\exp \left(-\frac{c}{<n>}\right)
\end{aligned}
$$

Indeed, if we expend and than integrate under this formula, we hind

$$
F(h)=\frac{c^{c}}{\Gamma(c)} \frac{\sigma}{<n>^{c}} \sum_{n \geq 2} h^{n} n^{c-1} \exp \left(-\frac{c}{<n>} n\right)
$$

which corresponds to the considered explicit form of the KNO function.

\section{Negative binomial distribution}

Negative binomial distribution (NBD) is defined as

$$
P(n)=\frac{\Gamma(n+r)}{n ! \Gamma(r)} p^{n}(1-p)^{r}, \sum_{n \geq 0} P(n)=1,
$$

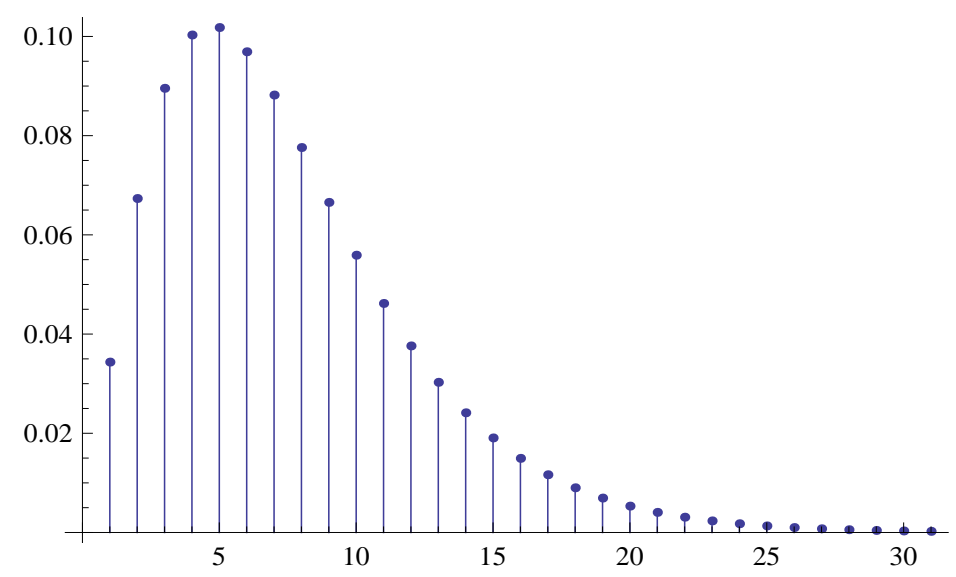

Figure 2: $P(n), r=2.8, p=0.3,<n>=6$

NBD provides a very good parametrization for multiplicity distributions in $e^{+} e^{-}$annihilation; in deep inelastic lepton scattering; in proton-proton collisions; in proton-nucleus scattering.

Hadronic collisions at high energies (LHC) lead to charged multiplicity distributions whose shapes are well fitted by a single NBD in fixed intervals of central (pseudo)rapidity $\eta$ [ALICE, 2010].

It is interesting to understand how NBD fits such a different reactions? 


\subsection{NBD and KNO scaling}

Let us consider NBD for normed topological cross sections

$$
\begin{aligned}
& \frac{\sigma_{n}}{\sigma}=P(n)=\frac{\Gamma(n+k)}{\Gamma(n+1) \Gamma(k)}\left(\frac{k}{<n>}\right)^{k}\left(1+\frac{k}{<n>}\right)^{-(n+k)} \\
& =\frac{\Gamma(n+k)}{\Gamma(n+1) \Gamma(k)}\left(1+\frac{k}{<n>}\right)^{-n}\left(1+\frac{<n>}{k}\right)^{-k} \\
& =\frac{\Gamma(n+k)}{\Gamma(n+1) \Gamma(k)}\left(\frac{<n>}{<n>+k}\right)^{n}\left(\frac{k}{k+<n>}\right)^{k}, \\
& =\frac{\Gamma(n+k)}{\Gamma(n+1) \Gamma(k)} \frac{\left(\frac{k}{<n>}\right)^{k}}{\left(1+\frac{k}{<n>}\right)^{k+n}}, \\
& r=k>0, p=\frac{<n>}{<n>+k} .
\end{aligned}
$$

The generating function for NBD is

$$
\begin{aligned}
& \left.F(h)=\left(1+\frac{<n>}{k}(1-h)\right)^{-k}=\left(1+\frac{<n>}{k}\right)^{-k}(1-a h)\right)^{-k}, \\
& a=p=\frac{<n>}{<n>+k} .
\end{aligned}
$$

Indeed,

$$
\begin{aligned}
& (1-a h))^{-k}=\frac{1}{\Gamma(k)} \int_{0}^{\infty} d t t^{k-1} e^{-t(1-a h)} \\
& =\frac{1}{\Gamma(k)} \int_{0}^{\infty} d t t^{k-1} e^{-t} \sum_{0}^{\infty} \frac{(t a h)^{n}}{n !} \\
& =\sum_{0}^{\infty} \frac{\Gamma(n+k) a^{n}}{\Gamma(k) n !} h^{n}, \\
& P(n)=\left(1+\frac{<n>}{k}\right)^{-k} \frac{\Gamma(n+k)}{\Gamma(k) n !}\left(\frac{<n>}{<n>+k}\right)^{n} \\
& =\frac{k^{k} \Gamma(n+k)}{\Gamma(k) \Gamma(n+1)}(<n>+k)^{-(n+k)}<n>^{n} \\
& =\frac{\Gamma(n+k)}{\Gamma(n+1) \Gamma(k)}\left(\frac{k}{<n>}\right)^{k}\left(1+\frac{k}{<n>}\right)^{-(n+k)}
\end{aligned}
$$

Note that KNO characteristic function ([6.13) coincides with the NBD generating function ([L.3) when $t=<n>(h-1), c=k$.

The Bose-Einstein distribution is a special case of NBD with $k=1$.

If $k$ is negative, the NBD becomes a positive binomial distribution, narrower than Poisson (corresponding to negative correlations).

For negative (integer) values of $k=-N$, we have Binomial GF

$$
\begin{aligned}
& F_{b d}=\left(1+\frac{<n>}{N}(h-1)\right)^{N}=(a+b h)^{N}, a=1-\frac{<n>}{N}, b=\frac{<n>}{N}, \\
& P_{b d}(n)=C_{N}^{n}\left(\frac{<n>}{N}\right)^{n}\left(1-\frac{<n>}{N}\right)^{N-n}
\end{aligned}
$$


(In a sense) we have a (quantum) spectrum for the parameter $k$, which contains any (positive) real values and (with finite number of states) the negative integer values, $(0 \leq n \leq N)$

From the generating function we have

$$
<n^{2}>=\left.\left(\frac{h d}{d h}\right)^{2} F(h)\right|_{h=1}=\frac{k+1}{k}<n>^{2}+<n>,
$$

for dispersion we obtain

$$
\begin{aligned}
& D=\sqrt{\left.<n^{2}>-<n\right\rangle^{2}}=\frac{1}{\sqrt{k}}<n>\left(1+\frac{k}{<n>}\right)^{1 / 2} \\
& =\frac{1}{\sqrt{k}}<n>+\frac{\sqrt{k}}{2}+O(1 /<n>),
\end{aligned}
$$

so the dispersion low for KNO and NBD distributions are the same, with $c=k$, for high values of the mean multiplicity.

The factorial moments of NBD,

$$
F_{m}=\left.\left(\frac{d}{d h}\right)^{m} F(h)\right|_{h=1}=\frac{<n(n-1) \ldots(n-m+1)>}{<n>^{m}}=\frac{\Gamma(m+k)}{\Gamma(m) k^{m}},
$$

and usual normalized moments of KNO (6.14) coincides.

\subsection{Fractal factorial and cumulant moments}

Using fractal calculus (see e.g. [Makhaldiani, 2003]),

$$
\begin{aligned}
D_{0, x}^{-\alpha} f & =\frac{|x|^{\alpha}}{\Gamma(\alpha)} \int_{0}^{1}|1-t|^{\alpha-1} f(x t) d t,=\frac{|x|^{\alpha}}{\Gamma(\alpha)} B(\alpha, \partial x) f(x) \\
& =|x|^{\alpha} \frac{\Gamma(\partial x)}{\Gamma(\alpha+\partial x)} f(x), f(x t)=t^{x \frac{d}{d x}} f(x) .
\end{aligned}
$$

we can define factorial and cumulant moments for any complex indexes,

$$
\begin{aligned}
& F_{-q}=<n>\left.^{q} D_{0, x}^{-q} G_{N B D}(x)\right|_{x=0}=\frac{k^{q} \Gamma(k-q)}{\Gamma(k)}, \\
& K_{-q}=<n>\left.^{q} D_{0, x}^{-q} \ln G_{N B D}(x)\right|_{x=0}=k^{q+1} \Gamma(-q), \\
& H_{-q}=\frac{\Gamma(k+1) \Gamma(-q)}{\Gamma(k-q)}
\end{aligned}
$$

\subsection{The KNO as asymptotic NBD}

Let us show that NBD is a discrete distribution corresponding to the KNO scaling,

$$
\lim _{<n>\rightarrow \infty}<n>\left.P_{n}\right|_{\frac{n}{<n>}=z}=\Psi(z)
$$

Indeed, using the following asymptotic formula

$$
\Gamma(x+1)=x^{x} e^{-x} \sqrt{2 \pi x}\left(1+\frac{1}{12 x}+O\left(x^{-2}\right)\right),
$$


we find

$$
\begin{aligned}
& <n>P_{n}=<n>\frac{(n+k-1)^{n+k-1} e^{-(n+k-1)}}{\Gamma(k) n^{n} e^{-n}} \frac{k^{k}}{n^{k}}<n>z^{k} e^{-k \frac{n+k}{<n>}} \\
& =\frac{k^{k}}{\Gamma(k)} z^{k-1} e^{-k z}+O(1 /<n>)
\end{aligned}
$$

We can calculate also $1 /<n>$ correction term to the KNO from the NBD. The answer is

$$
\Psi=\frac{k^{k}}{\Gamma(k)} z^{k-1} e^{-k z}\left(1+\frac{k^{2}}{2}\left(z-2+\frac{k-1}{k z}\right) \frac{1}{<n>}\right)
$$

This form coincides with the corrected KNO (G.2) for $c=k$ and $C_{2}^{1}=1$.

We have seen that KNO characteristic function (6.J3]) and NBD GF ([L.3) have almost same form. This relation become in coincidence if

$$
c=k, t=(h-1) \frac{<n>}{k}
$$

Now the definition of the characteristic function (6.13) can be read as

$$
\int_{0}^{\infty} e^{-<n>z(1-h)} \Psi(z) d z=\left(1+\frac{<n>}{k}(1-h)\right)^{-k}
$$

which means that Poisson GF weighted by KNO distribution gives NBD GF. Because of this, the NBD is the gamma-Poisson (mixture) distribution. This is the exact and universal picture of hadronization in multiparticle production processes.

\subsection{NBD, Poisson and Gauss distributions}

Fore high values of $x_{2}=k$ the NBD distribution reduces to the Poisson distribution

$$
\begin{aligned}
& F\left(x_{1}, x_{2}, h\right)=\left(1+\frac{x_{1}}{x_{2}}(1-h)\right)^{-x_{2}} \Rightarrow e^{-x_{1}(1-h)}=e^{-<n>} e^{h<n>} \\
& =\sum P(n) h^{n}, \\
& P(n)=e^{-<n>} \frac{<n>^{n}}{n !}
\end{aligned}
$$

For the Poisson distribution

$$
\begin{aligned}
& \left.\frac{d^{2} F(h)}{d h^{2}}\right|_{h=1}=<n(n-1)>=<n>^{2}, \\
& D^{2}=<n^{2}>-<n>^{2}=<n>.
\end{aligned}
$$

In the case of NBD, we had the following dispersion low

$$
D^{2}=\frac{1}{k}<n>^{2}+<n>,
$$

which coincides withe previous expression for high values of $k$.

Poisson GF belongs to the class of the infinitely divisible distributions,

$$
F(h,<n>)=(F(h,<n>/ k))^{k}
$$

For high values of $\langle n\rangle$, the Poisson distribution reduces to the Gauss distribution

$$
P(n)=e^{-<n>} \frac{<n>^{n}}{n !} \Rightarrow \frac{1}{\sqrt{2 \pi<n>}} \exp \left(-\frac{(n-<n>)^{2}}{2<n>}\right)
$$

For high values of $k$ in the integral relation (‥16), in the KNO function dominates the value $z_{c}=1$ and both sides of the relation reduce to the Poisson GF. 


\subsection{Multiplicative properties of KNO and NBD and corresponding motion equations}

An useful property of the negative binomial distribution with parameters

$$
<n>, k
$$

is that it is (also) the distribution of a sum of $k$ independent random variables drawn from a BoseEinstein distribution ${ }^{1}$ with mean $<n>/ k$,

$$
\begin{aligned}
& P_{n}=\frac{1}{<n>+1}\left(\frac{<n>}{<n>+1}\right)^{n} \\
& =\left(e^{\beta \hbar \omega / 2}-e^{-\beta \hbar \omega / 2}\right) e^{-\beta \hbar \omega(n+1 / 2)}, T=\frac{\hbar \omega}{\ln \frac{<n>+1}{<n>}} \\
& \sum_{n \geq 0} P_{n}=1, \sum_{n} n P_{n}=<n>=\frac{1}{e^{\beta \hbar \omega-1}}, T \simeq \hbar \omega<n>,<n>\gg 1, \\
& P(x)=\sum_{n} x^{n} P_{n}=(1+<n>(1-x))^{-1} .
\end{aligned}
$$

This is easily seen from the generating function in $(\mathbb{Z} .3])$, remembering that the generating function of a sum of independent random variables is the product of their generating functions.

Indeed, for

$$
n=n_{1}+n_{2}+\ldots+n_{k},
$$

with $n_{i}$ independent of each other, the probability distribution of $n$ is

$$
\begin{aligned}
& P_{n}=\sum_{n_{1}, \ldots, n_{k}} \delta\left(n-\sum n_{i}\right) p_{n_{1}} \ldots p_{n_{k}}, \\
& P(x)=\sum_{n} x^{n} P_{n}=p(x)^{k}
\end{aligned}
$$

This has a consequence that an incoherent superposition of $\mathrm{N}$ emitters that have a negative binomial distribution with parameters $k,\langle n>$ produces a negative binomial distribution with parameters $N k, N<n>$.

So, for the GF of NBD we have $(\mathrm{N}=2)$

$$
F(k,<n>) F(k,<n>)=F(2 k, 2<n>)
$$

And more general formula $(\mathrm{N}=\mathrm{m})$ is

$$
F(k,<n>)^{m}=F(m k, m<n>)
$$

We can put this equation in the closed nonlocal form

$$
Q_{q} F=F^{q},
$$

where

$$
Q_{q}=q^{D}, \quad D=\frac{k d}{d k}+\frac{<n>d}{d<n>}=\frac{x_{1} d}{d x_{1}}+\frac{x_{2} d}{d x_{2}}
$$

\footnotetext{
${ }^{1}$ A Bose-Einstein, or geometrical, distribution is a thermal distribution for single state systems.
} 
Note that temperature defined in (प22) gives an estimation of the Glukvar temperature when it radiates hadrons. If we take $\hbar \omega=100 \mathrm{MeV}$, to $T \simeq T_{c} \simeq 200 \mathrm{MeV}$ corresponds $<n>\simeq 1.5$ If we take $\hbar \omega=10 \mathrm{MeV}$, to $T \simeq T_{c} \simeq 200 \mathrm{MeV}$ corresponds $\langle n>\simeq 20$. A singular behavior of $\langle n>$ may indicate corresponding phase transition and temperature. At that point we estimate characteristic quant $\hbar \omega$.

We see that universality of NBD in hadron-production is similar to the universality of black body radiation.

\section{8. $p$-adic string theory}

p-adic string amplitudes can be obtained as tree amplitudes of the field theory with the following lagrangian and motion equation (see e.g. [Brekke, Freund, 1993])

$$
\begin{aligned}
& L=\frac{1}{2} \Phi Q_{p} \Phi-\frac{1}{p+1} \Phi^{p+1} \\
& Q_{p} \Phi=\Phi^{p}, Q_{p}=p^{D} \\
& D=-\frac{1}{2} \triangle, \triangle=-\partial_{x_{0}}^{2}+\partial_{x_{1}}^{2}+\ldots+\partial_{x_{n-1}}^{2}
\end{aligned}
$$

$\Phi$ - is real scalar field on $D$-dimensional space-time with coordinates $x=\left(x_{0}, x_{1}, \ldots, x_{D-1}\right)$. We have trivial, $\Phi=0$ and $\Phi=1$, and following nontrivial solutions of the equation (B.

$$
\Phi\left(x_{0}, x_{1}, \ldots, x_{D-1}\right)=p^{\frac{D}{2(p-1)}} e^{\frac{1-p^{-1}}{2 \ln p}\left(x_{0}^{2}-x_{1}^{2}-x_{2}^{2}-\ldots-x_{D-1}^{2}\right)}
$$

\section{1 p-adic solitons}

The equation (8. (ل) permits factorization of its solutions $\Phi(x)=\Phi\left(x_{0}\right) \Phi\left(x_{1}\right) \ldots \Phi\left(x_{D-1}\right)$, every factor of which fulfils one dimensional equation

$$
p^{\varepsilon \partial_{x}^{2}} \Phi(x)=\Phi(x)^{p}, \varepsilon= \pm \frac{1}{2}
$$

The trivial solution of the equations are $\Phi=0$ and $\Phi=1$. For nontrivial solution of (8. 4 ), we have

$$
\begin{aligned}
& p^{\varepsilon \partial_{x}^{2}} \Phi(x)=e^{a \partial^{2}} \Phi(x)=\frac{1}{\sqrt{4 \pi a}} \int_{-\infty}^{\infty} d y e^{-\frac{1}{4 a} y^{2}+y \partial} \Phi(x) \\
& =\frac{1}{\sqrt{4 \pi a}} \int_{-\infty}^{\infty} d y e^{-\frac{1}{4 a} y^{2}} \Phi(x+y)=\Phi(x)^{p}, a=\varepsilon \ln p
\end{aligned}
$$

If we (de quantize) put, $p=q$, and take (classical) limit, $q \rightarrow 1$, the motion equation reduce to

$$
\varepsilon \partial_{x}^{2} \Phi=\Phi \ln \Phi,
$$

with solution

$$
\Phi(x)=e^{\frac{1}{2}} e^{\frac{x^{2}}{4 \varepsilon}}
$$

It is obvious that the anzac

$$
\Phi=A e^{b x^{2}}
$$

can pass the equation (8.5)). Indeed, the solution is

$$
\begin{aligned}
& \Phi(x)=p^{\frac{1}{2(p-1)}} e^{\frac{1-p^{-1}}{4 \varepsilon \ln p} x^{2}}, \\
& \Phi\left(x_{0}, x_{1}, \ldots, x_{D-1}\right)=p^{\frac{D}{2(p-1)}} e^{\frac{1-p^{-1}}{2 \ln p}\left(x_{0}^{2}-x_{1}^{2}-x_{2}^{2}-\ldots-x_{D-1}^{2}\right)}
\end{aligned}
$$




\subsection{Corresponding class of the motion equations}

Now, we can define the following class of motion equations

$$
Q_{q} F=F^{q},
$$

where

$$
Q_{q}=q^{D}, D=D_{1}\left(x_{1}\right)+\ldots+D_{l}\left(x_{l}\right),
$$

$D_{k}(x)$ is some (differential) operator depending on $x$. In the case of the NBD GF,

$$
D_{k}(x)=\frac{x d}{d x}
$$

For this (Qlike) class of equations, we have factorization property

$$
\begin{aligned}
& F=F\left(x_{1}, \ldots, x_{l}\right)=F_{1}\left(x_{1}\right) \ldots F_{l}\left(x_{l}\right), \\
& q^{D_{k}(x)} F_{k}(x)=c_{k} F_{k}(x)^{q}, 1 \leq k \leq l, c_{1} c_{2} \ldots c_{l}=1 .
\end{aligned}
$$

\subsection{NBD motivated equations}

For NBD distribution we have corresponding multiplication(convolution)formulas

$$
\begin{aligned}
& (P \star P)_{n} \equiv \sum_{m=0}^{n} P_{m}(k,<n>) P_{n-m}(k,<n>) \\
& =P_{n}(2 k, 2<n>)=Q_{2} P_{n}(k,<n>), \ldots
\end{aligned}
$$

So, we can say, that star-product on the distributions of NBD corresponds ordinary product for GF.

It will be nice to have similar things for string field theory(SFT) [Kaku, 2000].

SFT motion equation is

$$
Q \Phi=\Phi \star \Phi
$$

For stringfield GF F we may have

$$
Q F=F^{2} .
$$

By construction we know the solution of the nice equation (L27) as GF of NBD, F. We obtain corresponding differential equations, if we consider $q=1+\varepsilon$, for small $\varepsilon$,

$$
\begin{aligned}
& \left(D(D-1) \ldots(D-m+1)-(\ln F)^{m}\right) \Psi=0, \\
& \left(\frac{\Gamma(D+1)}{\Gamma(D+1-m)}-(\ln F)^{m}\right) \Psi=0, \\
& \left(D_{m}-\Phi^{m}\right) \Psi=0, m=1,2,3, \ldots \\
& D_{m}=\frac{\Gamma(D+1)}{\Gamma(D+1-m)}, \Phi=\ln F
\end{aligned}
$$

with the solution $\Psi=F=\exp (\Phi)$. In the case of the NBD and p-adic string, we have correspondingly

$$
D=\frac{x_{1} d}{d x_{1}}+\frac{x_{2} d}{d x_{2}}
$$




$$
D=-\frac{1}{2} \triangle, \triangle=-\partial_{x_{0}}^{2}+\partial_{x_{1}}^{2}+\ldots+\partial_{x_{n-1}}^{2}
$$

These equations have meaning not only for integer $m$.

For high mean multiplicities we have corresponding equations for KNO

$$
\begin{aligned}
& Q_{2} \Psi(z)=\Psi \star \Psi \equiv \int_{0}^{z} \Psi(t) \Psi(z-t) d t \\
& =\left.z \int_{0}^{1} d t t^{\delta_{1}}(1-t)^{\delta_{2}} \Psi\left(z_{1}\right) \Psi\left(z_{2}\right)\right|_{z_{1}=z_{2}=z} \\
& =\left.z \frac{\Gamma\left(\delta_{1}+1\right) \Gamma\left(\delta_{2}+1\right)}{\Gamma\left(\delta_{1}+\delta_{2}+2\right)} \Psi\left(z_{1}\right) \Psi\left(z_{2}\right)\right|_{z_{1}=z_{2}=z}
\end{aligned}
$$

Due to the explicit form of the operator $D$, these equations and corresponding solutions have the symmetry under the change of the variables

$$
k \rightarrow a k,<n>\rightarrow b<n>.
$$

When

$$
a=\frac{<n>}{k}, b=\frac{k}{<n>},
$$

we obtain the symmetry with respect to the transformations $k \leftrightarrow<n>, x_{1} \leftrightarrow x_{2}$.

\section{Zeros of the Riemann zeta function}

The Riemann zeta function $\zeta(s)$ is defined for complex $s=\sigma+i t$ and $\sigma>1$ by the expansion

$$
\begin{aligned}
& \zeta(s)=\sum_{n \geq 1} n^{-s}, \operatorname{Re} s>1, \\
& =\left.\delta_{x}^{-s} \frac{x}{1-x}\right|_{x \rightarrow 1}=\left.\frac{1}{\Gamma(s)} \int_{0}^{\infty} t^{s-1} e^{-\delta_{x} t} \frac{x}{1-x}\right|_{x \rightarrow 1}=\left.\frac{1}{\Gamma(s)} \int_{0}^{\infty} t^{s-1} e^{t \partial_{\tau}} \frac{1}{e^{\tau}-1}\right|_{\tau \rightarrow 0} \\
& =\frac{1}{\Gamma(s)} \int_{0}^{\infty} \frac{t^{s-1} d t}{e^{t}-1}, x=e^{-\tau} .
\end{aligned}
$$

All complex zeros, $s=\alpha+i \beta$, of $\zeta(\sigma+i t)$ function lie in the critical stripe $0<\sigma<1$, symmetrically with respect to the real axe and critical line $\sigma=1 / 2$. So it is enough to investigate zeros with $\alpha \leq 1 / 2$ and $\beta>0$. These zeros are of three type, with small, intermediate and big ordinates.

\subsection{Riemann hypothesis}

The Riemann hypothesis [Titchmarsh, 1986] states that the (non-trivial) complex zeros of $\zeta(s)$ lie on the critical line $\sigma=1 / 2$.

At the beginning of the XX century Polya and Hilbert made a conjecture that the imaginary part of the Riemann zeros could be the oscillation frequencies of a physical system ( $\zeta$ - (mem)brane).

After the advent of Quantum Mechanics, the Polya-Hilbert conjecture was formulated as the existence of a self-adjoint operator whose spectrum contains the imaginary part of the Riemann zeros.

The Riemann hypothesis (RH) is a central problem in Pure Mathematics due to its connection with Number theory and other branches of Mathematics and Physics. 


\subsection{The functional equation for zeta function}

The functional equation is (see e.g. [Titchmarsh, 1986])

$$
\zeta(1-s)=\frac{2 \Gamma(s)}{(2 \pi)^{s}} \cos \left(\frac{\pi s}{2}\right) \zeta(s)
$$

From this equation we see the real (trivial) zeros of zeta function:

$$
\zeta(-2 n)=0, n=1,2, \ldots
$$

Also, at $\mathrm{s}=1$, zeta has pole with reside 1 .

From Field theory and statistical physics point of view, the functional equation (Q2.2) is duality relation, with self dual (or critical) line in the complex plane, at $s=1 / 2+i \beta$,

$$
\zeta\left(\frac{1}{2}-i \beta\right)=\frac{2 \Gamma(s)}{(2 \pi)^{s}} \cos \left(\frac{\pi s}{2}\right) \zeta\left(\frac{1}{2}+i \beta\right),
$$

we see that complex zeros lie symmetrically with respect to the real axe.

On the critical line, (nontrivial) zeros of zeta corresponds to the infinite value of the free energy,

$$
F=-T \ln \zeta
$$

At the point with $\beta=14.134725 \ldots$ is located the first zero. In the interval $10<\beta<100$, zeta has 29 zeros. The first few million zeros have been computed and all lie on the critical line. It has been proved that uncountably many zeros lie on critical line.

The first relation of zeta function with prime numbers is given by the following formula,

$$
\zeta(s)=\prod_{p}\left(1-p^{-s}\right)^{-1}, \operatorname{Re} s>1 .
$$

Another formula, which can be used on critical line, is

$$
\begin{aligned}
& \zeta(s)=\left(1-2^{1-s}\right)^{-1} \sum_{n \geq 1}(-1)^{n+1} n^{-s}, \text { Re } s>0 \\
& =\left.\frac{e^{i \pi\left(\delta_{x}+1\right)}}{\left(1-2^{1-s}\right) \delta_{x}^{s}} \frac{x}{1-x}\right|_{x \rightarrow 1}=\left.\frac{1}{1-2^{1-s}} \frac{1}{\Gamma(s)} \int_{0}^{\infty} d t t^{s-1} e^{i \pi} e^{(i \pi-t) \delta_{x}} \frac{1}{x^{-1}-1}\right|_{x \rightarrow 1} \\
& =\left.\frac{1}{1-2^{1-s}} \frac{1}{\Gamma(s)} \int_{0}^{\infty} d t t^{s-1} e^{(t-i \pi) \partial_{\tau}} \frac{e^{i \pi}}{e^{\tau}-1}\right|_{\tau \rightarrow 0}=\frac{1}{1-2^{1-s}} \frac{1}{\Gamma(s)} \int_{0}^{\infty} \frac{t^{s-1} d t}{e^{t}+1}, \\
& \int_{0}^{\infty} \frac{t^{s-1} d t}{e^{t}+1}=\int_{0}^{\infty} d t t^{s-1} e^{-t} \sum_{n \geq 0}(-1)^{n} e^{-n t}=\Gamma(s) \sum_{n \geq 1}(-1)^{n+1} n^{-s}
\end{aligned}
$$

\subsection{From Qlike to zeta equations}

Let us consider the values $q=n, n=1,2,3, \ldots$ and take sum of the corresponding equations (8.10), we find

$$
\zeta(-D) F=\frac{F}{1-F}
$$


In the case of the NBD we know the solutions of this equation.

Now we invent a Hamiltonian $H$ with spectrum corresponding to the set of nontrivial zeros of the zeta function, in correspondence with Riemann hypothesis,

$$
\begin{aligned}
& -D_{n}=\frac{n}{2}+i H_{n}, H_{n}=i\left(\frac{n}{2}+D_{n}\right), \\
& D_{n}=x_{1} \partial_{1}+x_{2} \partial_{2}+\ldots+x_{n} \partial_{n}, H_{n}^{+}=H_{n}=\sum_{m=1}^{n} H_{1}\left(x_{m}\right), \\
& H_{1}=i\left(\frac{1}{2}+x \partial_{x}\right)=-\frac{1}{2}(x \hat{p}+\hat{p} x), \hat{p}=-i \partial_{x}
\end{aligned}
$$

The Hamiltonian $H=H_{n}$ is hermitian, its spectrum is real. The case $n=1$ corresponds to the Riemann hypothesis.

The case $n=2$, corresponds to NBD,

$$
\begin{aligned}
& \zeta\left(1+i H_{2}\right) F=\frac{F}{1-F},\left.\zeta\left(1+i H_{2}\right)\right|_{F}=\frac{1}{1-F}, \\
& F\left(x_{1}, x_{2} ; h\right)=\left(1+\frac{x_{1}}{x_{2}}(1-h)\right)^{-x_{2}}
\end{aligned}
$$

Let us scale $x_{2} \rightarrow \lambda x_{2}$ and take $\lambda \rightarrow \infty$ in (Q.10), we obtain

$$
\begin{aligned}
& \zeta\left(\frac{1}{2}+i H_{1}(x)\right) e^{-(1-h) x}=\frac{1}{e^{(1-h) x}-1}, \\
& \frac{1}{\zeta\left(\frac{1}{2}+i H(x)\right)} \frac{1}{e^{\varepsilon x}-1}=e^{-\varepsilon x}, \\
& H(x)=i\left(\frac{1}{2}+x \partial_{x}\right)=-\frac{1}{2}(x \hat{p}+\hat{p} x), H^{+}=H, \varepsilon=1-h .
\end{aligned}
$$

Now we scale $x \rightarrow x y$, multiply the equation by $y^{s-1}$ and integrate

$$
\begin{aligned}
& \frac{1}{\zeta\left(\frac{1}{2}+i H(x)\right)} \int_{0}^{\infty} d y \frac{y^{s-1}}{e^{\varepsilon x y}-1}=\int_{0}^{\infty} d y e^{-\varepsilon x y} y^{s-1}=\frac{1}{(\varepsilon x)^{s}} \Gamma(s), \\
& \frac{1}{\zeta\left(\frac{1}{2}+i H(x)\right)} \int_{0}^{\infty} d y \frac{y^{s-1}}{e^{\varepsilon x y}-1}=\frac{1}{\zeta\left(\frac{1}{2}+i H(x)\right)} x^{-s} \varepsilon^{-s} \Gamma(s) \zeta(s),
\end{aligned}
$$

so

$$
\begin{aligned}
& \zeta\left(\frac{1}{2}+i H(x)\right) x^{-s}=\zeta(s) x^{-s} \Rightarrow H(x) \psi_{E}=E \psi_{E}, \\
& \psi_{E}=c x^{-s}, s=\frac{1}{2}+i E
\end{aligned}
$$

we have correct way and can return to the previous step $(Q \mathrm{CD})$ and take the following transformation

$$
\begin{aligned}
& \frac{1}{e^{\varepsilon x y}-1}=\frac{1}{2 \pi} \int_{-\infty+i c}^{\infty+i c} d E x^{-i E-1 / 2} \varphi(E, \varepsilon y), \\
& \varphi(E, \varepsilon y)=\int_{0}^{\infty} d x \frac{x^{i E-\frac{1}{2}}}{e^{\varepsilon x y}-1}=\frac{\Gamma\left(\frac{1}{2}+i E\right)}{(\varepsilon y)^{i E+1 / 2}} \zeta\left(\frac{1}{2}+i E\right), \\
& \frac{1}{2 \pi} \int_{-\infty+i c}^{\infty+i c} d E x^{-i E-1 / 2} \varphi(E, \varepsilon y) \frac{1}{\zeta(1 / 2+i E)}=e^{-\varepsilon x y}
\end{aligned}
$$


If we take the following formula

$$
\zeta(s)=\frac{1}{\Gamma(s)} \int_{0}^{\infty} \frac{t^{s-1} d t}{e^{t}-1}
$$

which says that $\zeta$ function is the Mellin transformation, we can find

$$
\Gamma\left(1+i H_{2}\right) \frac{F}{1-F}=\int_{0}^{\infty} \frac{d t / t}{e^{t}-1} F^{1 / t}
$$

or

$$
\begin{aligned}
& \Gamma\left(1+i H_{2}\right) \Phi=\int_{0}^{\infty} \frac{d t / t}{e^{t}-1}\left(\frac{\Phi}{1+\Phi}\right)^{1 / t} \\
& \Phi=\frac{F}{1-F}=\frac{1}{\left(1+\frac{x_{1}}{x_{2}}(1-h)\right)^{x_{2}}-1}
\end{aligned}
$$

We can obtain also the following equation with argument of $\zeta_{N}$ on critical axis

$$
\begin{aligned}
& \zeta_{N}\left(\frac{1}{2}+i H_{1}\left(x_{2}\right)\right) F\left(x_{1}, x_{2}, h\right)=\sum_{n=1}^{N} \frac{1}{\left(1+\frac{x_{1}}{n x_{2}}(1-h)\right)^{n x_{2}}} \\
& =\sum_{n=1}^{N} F\left(x_{1}, n x_{2}, h\right), \\
& \zeta_{N}\left(\frac{1}{2}+i H_{1}\left(x_{2}\right)\right) F\left(\lambda x_{1}, x_{2}, h\right)=\sum_{n=1}^{N} \frac{1}{\left(1+\frac{\lambda x_{1}}{n x_{2}}(1-h)\right)^{n x_{2}}} \\
& =\sum_{n=1}^{N} F\left(\lambda x_{1}, n x_{2}, h\right) \simeq N e^{-\lambda(1-h) x_{1}}, N \gg 1 .
\end{aligned}
$$

Let us calculate next therm in the $1 / \lambda$ expansion in the (2.10)

$$
\begin{aligned}
& F\left(x_{1}, \lambda x_{2}, h\right)=\left(1+\frac{\varepsilon x_{1}}{\lambda x_{2}}\right)^{-\lambda x_{2}}=e^{-\lambda x_{2} \ln \left(1+\varepsilon \frac{x_{1}}{\lambda x_{2}}\right)} \\
& =e^{-\varepsilon x_{1}} e^{\frac{\left(\varepsilon x_{1}\right)^{2}}{2 \lambda x_{2}}+O\left(\lambda^{-2}\right)}=e^{-\varepsilon x_{1}}\left(1+\frac{\left(\varepsilon x_{1}\right)^{2}}{2 \lambda x_{2}}+O\left(\lambda^{-2}\right)\right), \\
& \left(F^{-1}-1\right)^{-1}=\left(e^{\lambda x_{2} \ln \left(1+\varepsilon \frac{x_{1}}{\lambda x_{2}}\right)}-1\right)^{-1} \\
& =\frac{1}{e^{\varepsilon x_{1}}-1}\left(1+\frac{e^{\varepsilon x_{1}}}{e^{\varepsilon x_{1}}-1} \frac{\left(\varepsilon x_{1}\right)^{2}}{2 \lambda x_{2}}+O\left(\lambda^{-2}\right)\right)
\end{aligned}
$$

The zero order term, $\lambda^{0}$ we already considered. The next, $\lambda^{-1}$ order term gives the following relations

$$
\begin{aligned}
& \zeta\left(-\delta_{1}-\delta_{2}\right) \frac{x_{1}^{2}}{x_{2}} e^{-\varepsilon x_{1}}=\frac{1}{x_{2}} \zeta\left(1-\delta_{1}\right) x_{1}^{2} e^{-\varepsilon x_{1}}=\frac{x_{1}^{2} e^{\varepsilon x_{1}}}{x_{2}\left(e^{\varepsilon x_{1}}-1\right)^{2}}, \\
& \zeta(1-\delta) x^{2} e^{-\varepsilon x}=\frac{x^{2} e^{\varepsilon x}}{\left(e^{\varepsilon x}-1\right)^{2}}=x^{2} e^{-\varepsilon x}+O\left(e^{-2 \varepsilon x}\right) \\
& \zeta(1-\delta) \Psi=E \Psi+O\left(e^{-2 \varepsilon x}\right), \Psi=x^{2} e^{-\varepsilon x}, E=1 .
\end{aligned}
$$

There have been a number of approaches to understanding the Riemann hypothesis based on physics (for a comprehensive list see [Watkins]) 
According to the idea of Berry and Keating, [Berry, Keating, 1997] the real solutions $E_{n}$ of

$$
\zeta\left(\frac{1}{2}+i E_{n}\right)=0,
$$

are energy levels, eigenvalues of a quantum Hermitian operator (the Riemann operator) associated with the one-dimensional classical hyperbolic Hamiltonian

$$
H_{c}=x p
$$

where $x$ and $p$ are the conjugate coordinate and momentum.

They suggest a quantization condition generating Riemann zeros. This Hamiltonian breaks time-reversal invariance since $(x, p) \rightarrow(x,-p) \Rightarrow H \rightarrow-H$. The classical Hamiltonian $H=x p$ of linear dilation, i.e. multiplication in $x$ and contraction in $p$, gives the Hamiltonian equations:

$$
\begin{aligned}
& \dot{x}=x, \\
& \dot{p}=-p,
\end{aligned}
$$

with the solution

$$
\begin{aligned}
& x(t)=x_{0} e^{t} \\
& p(t)=p_{0} e^{-t}
\end{aligned}
$$

for any nonzero $E=x_{0} p_{0}=x(t) p(t)$ is hyperbola in phase space.

The system is quantized by considering the dilation operator in the $\mathrm{x}$ space

$$
H=\frac{1}{2}(x p+p x)=-i \hbar\left(\frac{1}{2}+x \partial_{x}\right),
$$

which is the simplest formally Hermitian operator corresponding to the classical Hamiltonian. The eigenvalue equation

$$
H \psi_{E}=E \psi_{E}
$$

is satisfied by the eigenfunctions

$$
\psi_{E}(x)=c x^{-\frac{1}{2}+\frac{i}{\hbar} E},
$$

where the complex constant $c$ is arbitrary, since the solutions are not square-integrable. To the normalization

$$
\int_{0}^{\infty} d x \psi_{E}(x)^{*} \psi_{E^{\prime}}(x)=\delta\left(E-E^{\prime}\right)
$$

corresponds $c=1 / \sqrt{2 \pi}$.

We have seen that

$$
\begin{aligned}
& \zeta\left(\frac{1}{2}+i H\right) e^{-\varepsilon x}=\frac{1}{e^{\varepsilon x}-1}, \\
& H=-i\left(\frac{1}{2}+x \partial_{x}\right)=x^{1 / 2} p x^{1 / 2}, p=-i \partial_{x},
\end{aligned}
$$


than

$$
\begin{aligned}
& e^{-\varepsilon x}=\int d E x^{-1 / 2+i E} \varphi(E, \varepsilon), \\
& \varphi(E, \varepsilon)=\frac{1}{2 \pi} \int_{0}^{\infty} d x x^{-1 / 2-i E} e^{-\varepsilon x}=\frac{\varepsilon^{-1 / 2+i E}}{2 \pi} \Gamma(1 / 2-i E) \\
& \zeta\left(\frac{1}{2}+i E\right) \varphi(E, \varepsilon)=\frac{1}{2 \pi} \int_{0}^{\infty} d x \frac{x^{-1 / 2-i E}}{e^{\varepsilon x}-1} \\
& =\frac{\varepsilon^{-1 / 2+i E}}{2 \pi} \Gamma(1 / 2-i E) \zeta\left(\frac{1}{2}-i E\right) .
\end{aligned}
$$

\subsection{Some calculations with zeta function values}

From the equation (ㅁ] ) we have

$$
\begin{aligned}
& \zeta\left(\frac{1}{2}+i H_{1}(x)\right) e^{-\varepsilon x}=\frac{1}{e^{\varepsilon x}-1}, H_{1}=i\left(\frac{1}{2}+x \partial_{x}\right) \\
& \zeta\left(-x \partial_{x}\right)\left(1-\varepsilon x+\frac{(\varepsilon x)^{2}}{2}+\ldots\right)=\frac{1}{\varepsilon x}\left(1-\left(\frac{\varepsilon x}{2}+\frac{(\varepsilon x)^{2}}{6}+\ldots\right)+\right. \\
& \left.+\left(\frac{\varepsilon x}{2}+\ldots\right)^{2}+\ldots\right)
\end{aligned}
$$

so

$$
\zeta(0)=-\frac{1}{2}, \zeta(-1)=-\frac{1}{12}, \ldots
$$

Note that, a little calculation shows that, the $(\varepsilon x)^{2}$ terms cancels on the r.h.s, in accordance with $\zeta(-2)=0$.

More curious question concerns with the term $1 / \varepsilon x$ on the r.h.s. To it corresponds the term with actual infinitesimal coefficient on the 1.h.s.

$$
\frac{1}{\zeta(1)} \frac{1}{\varepsilon x}
$$

in the spirit of the nonstandard analysis (see, e.g. [Davis, 1977]), we can imagine that such a terms always present but on the r.h.s we may not note them.

For other values of zeta function we will use the following expansion

$$
\begin{aligned}
& \frac{1}{e^{x}-1}=\frac{1}{x+\frac{x^{2}}{2}+\frac{x^{3}}{3 !}+\ldots}=\frac{1}{x}-\frac{1}{2}+\sum_{k \geq 1} \frac{B_{2 k} x^{2 k-1}}{(2 k) !} \\
& B_{2}=\frac{1}{6}, B_{4}=-\frac{1}{30}, B_{6}=\frac{1}{42}, \ldots
\end{aligned}
$$

and obtain

$$
\zeta(1-2 n)=-\frac{B_{2 n}}{2 n}, n \geq 1
$$

\section{Multiparticle production stochastic dynamics}

Let us imagine space-time development of the the multiparticle process and try to describe it by some (phenomenological) dynamical equation. We start to find the equation for the Poisson distribution and than naturally extend them for the NBD case. 
Let us define an integer valued variable $n(t)$ as a number of events (produced particles) at the time $t, n(0)=0$. The probability of event $n(t), P(t, n)$, is defined from the following motion equation

$$
\begin{aligned}
& P_{t} \equiv \frac{\partial P(t, n)}{\partial t}=r(P(t, n-1)-P(t, n)), n \geq 1 \\
& \left.P_{t}(t, 0)\right)=-r P(t, 0), \\
& P(t, n)=0, n<0
\end{aligned}
$$

so

$$
\begin{aligned}
& P(t, 0) \equiv P_{0}(t)=e^{-r t}, \\
& P(t, n)=Q(t, n) P_{0}(t), \\
& Q_{t}(t, n)=r Q(t, n-1), Q(t, 0)=1 .
\end{aligned}
$$

To solve the equation for $Q$, we invent its generating function

$$
F(t, h)=\sum_{n \geq 0} h^{n} Q(t, n),
$$

and solve corresponding equation

$$
F_{t}=r h F, F(t, h)=e^{r t h}=\sum h^{n} \frac{(r t)^{n}}{n !}, Q(t, n)=\frac{(r t)^{n}}{n !},
$$

SO

$$
P(t, n)=e^{-r t} \frac{(r t)^{n}}{n !}
$$

is the Poisson distribution.

If we compare this distribution with (ㅍ.2]), we identify $\langle n\rangle=r t$, as if we have a free particle motion with velocity $r$ and the distance is the mean multiplicity. This way we have a connection between n-dimension of the multiplicity and the usual dimension of trajectory.

As the equation gives right solution, its generalization may give more general distribution, so we will generalize the equation (메). For this, we put the equation in the closed form

$$
\begin{aligned}
& P_{t}(t, n)=r\left(e^{-\partial_{n}}-1\right) P(t, n) \\
& =\sum_{k \geq 1} D_{k} \partial^{k} P(t, n), D_{k}=(-1)^{k} \frac{r}{k !},
\end{aligned}
$$

where the $D_{k}, k \geq 1$, are generalized diffusion coefficients.

For other values of the coefficients, we will have other distributions.

\subsection{Fractal dimension of the multiparticle production trajectories}

For mean square deviation of the trajectory we have

$$
<(x-\bar{x})^{2}>=<x^{2}>-<x>^{2} \equiv D(x)^{2} \sim t^{2 / d_{f}},
$$


where $d_{f}$ is fractal dimension. For smooth classical trajectory of particles we have $d_{f}=1$; for free stochastic, Brownian, trajectory, all diffusion coefficients are zero but $D_{2}$, we have $d_{f}=2$. In the case of Poisson process we have,

$$
D(n)^{2}=<n^{2}>-<n>^{2} \sim t, d_{f}=2 .
$$

In the case of the NBD and KNO distributions

$$
D(n)^{2} \sim t^{2}, d_{f}=1 .
$$

As we have seen, rasing $k$, KNO reduce to the Poisson, so we have a dimensional (phase) transition from the phase with dimension 1 to the phase with dimension 2. It is interesting, if somehow this phase transition is connected to the other phase transitions in strong interaction processes.

For the Poisson distribution GF is solution of the following equation,

$$
\dot{F}=-r(1-h) F,
$$

For the NBD corresponding equation is

$$
\dot{F}=\frac{-r(1-h)}{1+\frac{r t}{k}(1-h)} F=-R(t) F, R(t)=\frac{r(1-h)}{1+\frac{r t}{k}(1-h)} .
$$

If we change the time variable as $t=T^{d_{f}}$, we reduce the dispersion low from general fractal to the NBD like case. Corresponding transformation for the evolution equation is

$$
F_{T}=-d_{f} T^{d_{f}-1} R\left(T^{d_{F}}\right) F
$$

we ask that this equation coincides with NBD motion equation, and define rate function $R(T)$

$$
d_{f} T^{d_{f}-1} R\left(T^{d_{F}}\right)=\frac{r(1-h)}{1+\frac{r T}{k}(1-h)},
$$

now the following equation defines a production processes with fractal dimension $d_{F}$

$$
F_{t}=-R(t) F, R(t)=\frac{r(1-h)}{d_{F} t^{\frac{d_{F}-1}{d_{F}}}\left(1+\frac{r t^{1 / d_{F}}}{k}(1-h)\right)}
$$

\section{Spherical model of the multiparticle production}

Now we would like to consider a model of multiparticle production based on the d-dimensional sphere, and (try to) motivate the values of the NBD parameter $k$. The volum of the d-dimensional sphere with radius $r$, in units of hadron size $r_{h}$ is

$$
v(d, r)=\frac{\pi^{d / 2}}{\Gamma(d / 2+1)}\left(\frac{r}{r_{h}}\right)^{d}
$$

Note that,

$$
v(0, r)=1, v(1, r)=2 \frac{r}{r_{h}},
$$




$$
v(-1, r)=\frac{1}{\pi} \frac{r_{h}}{r}
$$

If we identify this dimensionless quantity with corresponding coulomb energy formula,

$$
\frac{1}{\pi}=\frac{e^{2}}{4 \pi}
$$

we find $e= \pm 2$.

For less then -1 even integer values of $d$, and $r \neq 0, v=0$. For negative odd integer $d=-2 n+1$

$$
\begin{aligned}
& v(-2 n+1, r)=\frac{\pi^{-n+1 / 2}}{\Gamma(-n+3 / 2)}\left(\frac{r_{h}}{r}\right)^{2 n-1}, n \geq 1, \\
& v(-3, r)=-\frac{1}{2 \pi^{2}}\left(\frac{r_{h}}{r}\right)^{3}, v(-5, r)=\frac{3}{4 \pi^{3}}\left(\frac{r_{h}}{r}\right)^{5}
\end{aligned}
$$

Note that,

$$
v(2, r) v(3, r) v(-5, r)=\frac{1}{\pi}, v(1, r) v(2, r) v(-3, r)=-\frac{1}{\pi}
$$

We postulate that after collision, it appear intermediate state with almost spherical form and constant energy density. Than the radius of the sphere rise, dimension decrease, volume remains constant. At the last moment of the expansion, when the crossection of the one dimensional sphere - string become of order of hadron size, hadronic string divide in $k$ independent sectors which start to radiate hadrons with geometric (Boze-Einstein) distribution, so all of the string final state radiate according to the NBD distribution.

So, from the volume of the hadronic string,

$$
v=\pi\left(\frac{r}{r_{h}}\right)^{2} \frac{l}{r_{h}}=\pi k
$$

we find the NBD parameter $k$,

$$
k=\frac{\pi^{d / 2-1}}{\Gamma(d / 2+1)}\left(\frac{r}{r_{h}}\right)^{d}
$$

Knowing, from experimental date, the parameter $k$, we can restrict the region of the values of the parameters $d$ and $r$ of the primordial sphere (PS),

$$
\begin{aligned}
& r(d)=\left(\frac{\Gamma(d / 2+1)}{\pi^{d / 2}-1} k\right)^{1 / d} r_{h}, \\
& r(3)=\left(\frac{3}{4} k\right)^{1 / 3} r_{h}, r(2)=k^{1 / 2} r_{h}, r(1)=\frac{\pi}{2} k r_{h}
\end{aligned}
$$

If the value of $r(d)$ will be a few $r_{h}$, the matter in the PS will be in the hadronic phase. If the value of $r$ will be of order $10 r_{h}$, we can speak about deconfined, quark-gluon, Glukvar, phase. From the formula (ए.9), we see, that to have for the $r$, the value of order $10 r_{h}$, in $d=3$ dimension, we need the value for $k$ of order 1000, which is not realistic. 
So in our model, we need to consider the lower than one, fractal, dimensions. It is consistent with the following intuitive picture. Confined matter have point-like geometry, with dimension zero. Primordial sphere of Glukvar have nonzero fractal dimension, which is less than one,

$$
\begin{aligned}
& k=3, r(0.7395) / r_{h}=10.00 \\
& k=4, r(0.8384) / r_{h}=10.00
\end{aligned}
$$

From the experimental data we find the parameter $k$ of the NBD as a function of energy, $k=k(s)$. Then, by our spherical model, we construct fractal dimension of the Glukvar as a function of $k(s)$.

If we suppose that radius of the primordial sphere $r$ is of order (or less) of $r_{h}$. Than we will have higher dimensional PS, e.g.

\begin{tabular}{|c|c|c|}
\hline $\mathrm{d}$ & $r / r_{h}$ & $\mathrm{k}$ \\
\hline 3 & 1.3104 & 3.0002 \\
4 & 1.1756 & 3.0003 \\
6 & 1.1053 & 2.9994 \\
8 & 1.1517 & 3.9990 \\
\hline
\end{tabular}

\subsection{Extra dimension effects at high energy and large scale Universe}

With extra dimensions gravitation interactions may become strong at the LHC energies,

$$
V(r)=\frac{m_{1} m_{2}}{m^{2+d}} \frac{1}{r^{1+d}}
$$

If the extra dimensions are compactified with(in) size $\mathrm{R}$, at $r>>R$,

$$
V(r) \simeq \frac{m_{1} m_{2}}{m^{2}(m R)^{d}} \frac{1}{r}=\frac{m_{1} m_{2}}{M_{P l}^{2}} \frac{1}{r},
$$

where (4-dimensional) Planck mass is given by

$$
M_{P l}^{2}=m^{2+d} R^{d},
$$

so the scale of extra dimensions is given as

$$
R=\frac{1}{m}\left(\frac{M_{P l}}{m}\right)^{\frac{2}{d}}
$$

If we take $m=1 T e V,\left(\mathrm{GeV}^{-1}=0.2 \mathrm{fm}\right)$

$$
\begin{aligned}
& R(d)=2 \cdot 10^{-17}\left(\frac{M_{P l}}{1 T e V}\right)^{\frac{2}{d}} \mathrm{~cm}, \\
& R(1)=2 \cdot 10^{15} \mathrm{~cm}, \\
& R(2)=0.2 \mathrm{~cm} ! \\
& R(3)=10^{-7} \mathrm{~cm} ! \\
& R(4)=2 \cdot 10^{-9} \mathrm{~cm}, \\
& R(6) \sim 10^{-11} \mathrm{~cm}
\end{aligned}
$$

Note that lab measurements of $G_{N}\left(=1 / M_{P l}^{2}, M_{P l}=1.2 \times 10^{19} \mathrm{GeV}\right)$ have been made only on scales of about $1 \mathrm{~cm}$ to $1 \mathrm{~m}$; 1 astronomical unit(AU) (mean distance between Sun and Earth) is $1.5 \times$ 
$10^{13} \mathrm{~cm}$; the scale of the periodic structure of the Universe, $L=128 \mathrm{Mps} \simeq 4 \cdot 10^{26} \mathrm{~cm}$. It is curious which (small) value of the extra dimension corresponds to $L$ ?

$$
\begin{aligned}
d & =2 \frac{\ln \frac{M_{P l}}{m}}{\ln (m L)}=0.74, m=1 \mathrm{TeV}, \\
& =0.81, m=100 \mathrm{GeV} \\
& =0.07, m=10^{17} \mathrm{GeV} .
\end{aligned}
$$

\section{Dynamical formulation of $\mathrm{z}$ - Scaling}

Motion equations of physics (applied mathematics in general) connect different observable quantities and reduce the number of independently measurable quantities. More fundamental equation contains less number of independent quantities. When (before) we solve the equations, we invent dimensionless invariant variables, than one solution can describe all of the class of phenomena.

In the $\mathrm{z}$ - Scaling $(\mathrm{zS})$ approach to the inclusive multiparticle distributions (MPD) (see, e.g. [Tokarev, Zborovsky, 2007]), different inclusive distributions depending on the variables $x_{1}, \ldots x_{n}$, are described by universal function $\Psi(z)$ of fractal variable $z$,

$$
z=x_{1}^{-\alpha_{1}} \ldots x_{n}^{-\alpha_{n}} .
$$

It is interesting to find a dynamical system which generates this distributions and describes corresponding MPD.

We can find a good function if we know its derivative. Let us consider the following RD like equation

$$
\begin{aligned}
& z \frac{d}{d z} \Psi=V(\Psi) \\
& \int_{\Psi\left(z_{0}\right)}^{\Psi(z)} \frac{d x}{V(x)}=\ln \frac{z}{z_{0}}
\end{aligned}
$$

In $x$-representation,

$$
\begin{aligned}
& \ln z=-\sum_{k=1}^{n} \alpha_{k} \ln x_{k}, \delta_{z}=z \frac{d}{d z}=-\sum_{k} \frac{\delta_{k}}{n_{h} \alpha_{k}} \\
& \sum_{k=1}^{n} \frac{x_{k}}{n_{h} \alpha_{k}} \frac{\partial}{\partial x_{k}} \Psi\left(x_{1}, \ldots, x_{n}\right)+V(\Psi)=0
\end{aligned}
$$

e.g.

$$
z=\delta_{z} z=-\sum_{k=1}^{n} \frac{x_{k}}{n_{h} \alpha_{k}} \frac{\partial}{\partial x_{k}} x_{1}^{-\alpha_{1}} \ldots x_{n}^{-\alpha_{n}}=z, n_{h}=n
$$

In the case of NBD GF ([.27), we have

$$
\begin{aligned}
& n=2, x_{1}=k, x_{2}=<n>, \alpha_{1}=\alpha_{2}=1, n_{h}=1 \\
& \Psi=F, V(\Psi)=-\Psi \ln \Psi .
\end{aligned}
$$


In the case of the $z$-scaling, [Tokarev, Zborovsky, 2007],

$$
\begin{aligned}
& n=4, x_{3}=y_{a}, x_{4}=y_{b}, \\
& \alpha_{1}=\delta_{1}, \alpha_{2}=\delta_{2}, \alpha_{3}=\varepsilon_{a}, \alpha_{4}=\varepsilon_{b}, n_{h}=4,
\end{aligned}
$$

for infinite resolution, $\alpha_{n}=1, n=1,2,3,4$. In $z$ variable the equation for $\Psi$ has universal form. In the case of $n=2, \alpha_{1}=\alpha_{2}=1, n_{h}=1$, we find that $V(\Psi)=-\Psi \ln \Psi$,

$$
\begin{aligned}
& z \frac{d}{d z} \Psi(z)=-\Psi \ln \Psi, \\
& \Psi(z)=e^{c / z}=\left(\Psi\left(z_{0}\right)^{z_{0}}\right)^{\frac{1}{z}}=\Psi\left(z_{0}\right)^{\frac{z_{0}}{z}}, \\
& c=z_{0} \ln \Psi\left(z_{0}\right)<0, z \in(0, \infty), \Psi(z) \in(0,1) .
\end{aligned}
$$

Note that the fundamental equation is invariant with respect to the scale transformation $z \rightarrow \lambda z$, but the solution is not, the scale transformation transforms one solution into another solution. This is an example of the spontaneous breaking of the (scale) symmetry by the states of the system.

\subsection{Formal motivation (foundation) of the RD motion equation for $\Psi$}

As a dimensionless physical quantity $\Psi(z)$ may depend only on the running coupling constant $g(\tau), \tau=\ln z / z_{0}$

$$
\begin{aligned}
& z \frac{d}{d z} \Psi=\dot{\Psi}=\frac{d \Psi}{d g} \beta(g)=U(g)=U\left(f^{-1}(\Psi)\right)=V(\Psi) \\
& \Psi(\tau)=f(g(\tau)), g=f^{-1}(\Psi(\tau))
\end{aligned}
$$

\subsection{Realistic solution for $\Psi$}

According to the paper [Tokarev, Zborovsky, 2007], for high values of $z, \Psi(z) \sim z^{-\beta}$; for small $z, \Psi(z) \sim$ const.

So, for high $z$,

$$
z \frac{d}{d z} \Psi=V(\Psi(z))=-\beta \Psi(z)
$$

for smaller values of $z, \Psi(z)$ rise and we expect nonlinear terms in $V(\Psi)$,

$$
V(\Psi)=-\beta \Psi+\gamma \Psi^{2}
$$

With this function, we can solve the equation for $\Psi$ and find

$$
\Psi(z)=\frac{1}{\frac{\gamma}{\beta}+c z^{\beta}} .
$$

\subsection{Reparametrization of the RD equation}

$\mathrm{RD}$ equation of the $\mathrm{z}$-Scaling,

$$
z \frac{d}{d z} \Psi(z)=V(\Psi), V(\Psi)=V_{1} \Psi+V_{2} \Psi^{2}+\ldots+V_{n} \Psi^{n}+\ldots
$$


can be reparametrized,

$$
\begin{aligned}
& \Psi(z)=f(\psi(z))=\psi(z)+f_{2} \psi^{2}+\ldots+f_{n} \psi^{n}+\ldots \\
& z \frac{d}{d z} \psi(z)=v(z)=v_{1} \psi(z)+v_{2} \psi^{2}+\ldots+v_{n} \psi^{n}+\ldots \\
& \left(v_{1} \psi(z)+v_{2} \psi^{2}+\ldots+v_{n} \psi^{n}+\ldots\right)\left(1+2 f_{2} \psi+\ldots+n f_{n} \psi^{n-1}+\ldots\right) \\
& =V_{1}\left(\psi+f_{2} \psi^{2}+\ldots+f_{n} \psi^{n}+\ldots\right) \\
& +V_{2}\left(\psi^{2}+2 f_{2} \psi^{3}+\ldots\right)+\ldots+V_{n}\left(\psi^{n}+n f_{2} \psi^{n+1}+\ldots\right)+\ldots \\
& =V_{1} \psi+\left(V_{2}+V_{1} f_{2}\right) \psi^{2}+\left(V_{3}+2 V_{2} f_{2}+V_{1} f_{3}\right) \psi^{3}+ \\
& \ldots+\left(V_{n}+(n-1) V_{n-1} f_{2}+\ldots+V_{1} f_{n}\right) \psi^{n}+\ldots \\
& v_{1}=V_{1} \\
& v_{2}=V_{2}-f_{2} V_{1}, \\
& v_{3}=V_{3}+2 V_{2} f_{2}+V_{1} f_{3}-2 f_{2} v_{2}-3 f_{3} v_{1}=V_{3}+2\left(f_{2}^{2}-f_{3}\right) V_{1}, \ldots \\
& v_{n}=V_{n}+(n-1) V_{n-1} f_{2}+\ldots+V_{1} f_{n}-2 f_{2} v_{n-1}-\ldots-n f_{n} v_{1},
\end{aligned}
$$

so, by reparametrization, we can change any coefficient of potential $\mathrm{V}$ but $V_{1}$.

We can fix any higher coefficient with zero value, if we take

$$
\begin{aligned}
f_{2} & =\frac{V_{2}}{V_{1}}, f_{3}=\frac{V_{3}}{2 V_{1}}+f_{2}^{2}=\frac{V_{3}}{2 V_{1}}+\left({\frac{V_{2}}{V_{1}}}^{2}\right), \ldots \\
f_{n} & =\frac{V_{n}+(n-1) V_{n-1} f_{2}+\ldots+2 V_{2} f_{n-1}}{(n-1) V_{1}}, \ldots
\end{aligned}
$$

We will consider the case when only one of higher coefficient is nonzero and give explicit form of the solution $\Psi$.

\subsection{More general solution for $\Psi$}

Let us consider more general potential $V$

$$
z \frac{d}{d z} \Psi=V(\Psi)=-\beta \Psi(z)+\gamma \Psi(z)^{1+n}
$$

Corresponding solution for $\Psi$ is

$$
\Psi(z)=\frac{1}{\left(\frac{\gamma}{\beta}+c z^{n \beta}\right)^{\frac{1}{n}}}
$$

More general solution contains three parameters and may better describe the data of inclusive distributions.

In the case of $n=1$ we reproduce the previous solution.

Another "natural" case is $n=1 / \beta$,

$$
\Psi(z)=\frac{1}{\left(\frac{\gamma}{\beta}+c z\right)^{\beta}}
$$

In this case, we can absorb (interpret) the combined parameter by shift and scaling

$$
z \rightarrow \frac{1}{c}\left(z-\frac{\gamma}{\beta}\right)
$$




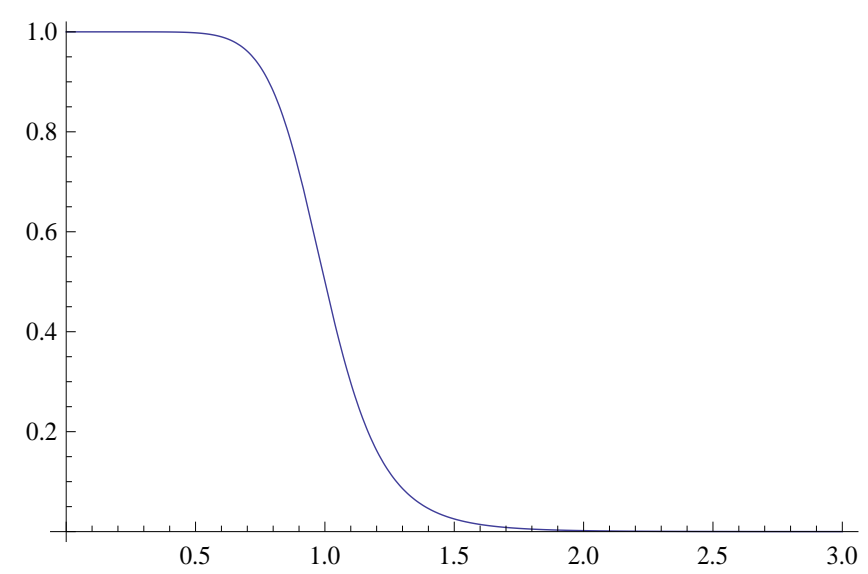

Figure 3: z-scaling distribution $\Psi(z, 9,9,1,1)$

Another interesting point of view is to predict the value of $\beta$

$$
\beta=\frac{1}{n}=0.5 ; 0.33 ; 0.25 ; 0.2 ; \ldots, n=2,3,4,5, \ldots
$$

For experimentally suggested value $\beta \simeq 9, n=0.11$

In the case of $n=-\varepsilon, \beta=\gamma=1 / \varepsilon, c=\varepsilon k$, we will have

$$
V(\Psi)=-\Psi \ln \Psi, \Psi(z)=e^{\frac{k}{z}}
$$

This form of $\Psi$-function interpolates between asymptotic values of $\Psi$ and predicts its behavior in the intermediate region.

The three parameter function is restricted by the normalization condition

$$
\begin{aligned}
& \int_{0}^{\infty} \Psi(z) d z=1, \\
& B\left(\frac{\beta-1}{\beta n}, \frac{1}{\beta n}\right)=\left(\frac{\beta}{\gamma}\right)^{\frac{\beta-1}{\beta n}} \frac{\beta n}{c^{\beta n}},
\end{aligned}
$$

so remains only two free parameter. When $\beta n=1$, we have

$$
c=(\beta-1)\left(\frac{\beta}{\gamma}\right)^{\beta-1}
$$

If $\beta n=1$ and $\beta=\gamma$, than $c=\beta-1$.

In general

$$
c^{\beta n}=\left(\frac{\beta}{\gamma}\right)^{\frac{\beta-1}{\beta n}} \frac{\beta n}{B\left(\frac{\beta-1}{\beta n}, \frac{1}{\beta n}\right)}
$$

\subsection{Scaling properties of scaling functions and they equations}

RD equation of the z-scaling (12.15), after substitution,

$$
\Psi(z)=(\varphi(z))^{\frac{1}{n}},
$$


reduce to the $n=1$ case with scaled parameters,

$$
\dot{\varphi}=-\beta n \varphi+\gamma n \varphi^{2}
$$

this substitution could be motivated also by the structure of the solution (12.16),

$$
\Psi(z, \beta, \gamma, n, c)=\Psi(z, \beta n, \gamma n, 1, c)^{\frac{1}{n}}=\Psi(z, \beta, \gamma, \beta n, c)^{\beta} .
$$

General RD equation takes form

$$
\dot{\varphi}=n v_{1} \varphi+n v_{2} \varphi^{1+\frac{1}{n}}+n v_{3} \varphi^{1+\frac{2}{n}}+\ldots+n v_{n} \varphi^{2}+n v_{n+1} \varphi^{2+\frac{1}{n}}+\ldots
$$

\subsection{Space-time dimension inside hadrons and nuclei}

The dimension of the space(-time) is the model dependent concept. E.g. for the fundamental bosonic string model (in flat space-time) the dimension is 26; for superstring model the dimension is 10 [Kaku, 2000].

Let us imagine that we have some action-functional formulation with the fundamental motion equation

$$
z \frac{d}{d z} \Psi=V(\Psi(z))=V(\Psi)=-\beta \Psi+\gamma \Psi^{1+n}
$$

Than, the corresponding Lagrangian contains the following mass and interaction parts

$$
-\frac{\beta}{2} \Psi^{2}+\frac{\gamma}{2+n} \Psi^{2+n}
$$

The action gives renormalizable (effective quantum field theory) model when

$$
d+2=\frac{2 N}{N-2}=\frac{2(2+n)}{n}=2+\frac{4}{n}=2+4 \beta,
$$

so, measuring the parameter $\beta$ inside hadronic and nuclear matters, we find corresponding (fractal) dimension.

\subsection{Another action formulation of the Fundamental equation}

From fundamental equation we obtain

$$
\begin{aligned}
& \left(z \frac{d}{d z}\right)^{2} \Psi \equiv \ddot{\Psi}=V^{\prime}(\Psi) V(\Psi)=\frac{1}{2}\left(V^{2}\right)^{\prime} \\
& =\beta^{2} \Psi-\beta \gamma(n+2) \Psi^{n+1}+\gamma^{2}(n+1) \Psi^{2 n+1}
\end{aligned}
$$

Corresponding action Lagrangian is

$$
\begin{aligned}
& L=\frac{1}{2} \dot{\Psi}^{2}+U(\Psi), U=\frac{1}{2} V^{2}=\frac{1}{2} \Psi^{2}\left(\beta-\gamma \Psi^{n}\right)^{2} \\
& =\frac{\beta^{2}}{2} \Psi^{2}-\beta \gamma \Psi^{2+n}+\frac{\gamma^{2}}{2} \Psi^{2+2 n}
\end{aligned}
$$

This potential, $-U$, has two maximums, when $V=0$, and minimum, when $V^{\prime}=0$, at $\Psi=0$ and $\Psi=(\beta / \gamma)^{1 / n}$, and $\Psi=(\beta /(n+1) \gamma)^{1 / n}$, correspondingly. 
We define time-space-scale field $\Psi(t, x, \eta)$, where $\eta=\ln z$ - is scale coordinate variable, with corresponding action functional

$$
A=\int d t d^{d} x d \eta\left(\frac{1}{2} g^{a b} \partial_{a} \Psi \partial_{b} \Psi+U(\Psi)\right)
$$

The renormalization constraint for this action is

$$
N=2+2 n=\frac{2(2+d)}{2+d-2}=2+\frac{4}{d}, d n=2, d=2 / n=2 \beta .
$$

So we have two models for spase-time dimension, (12.30) and (⒉34),

$$
d_{1}=4 \beta ; d_{2}=2 \beta
$$

The coordinate $\eta$ characterise (multiparticle production) physical process at the (external) spacetime point $(x, t)$. The dimension of the space-time inside hadrons and nuclei, where multiparticle production takes place is

$$
d+1=1+2 \beta
$$

Note that this formula reminds the dimension of the spin s state, $d_{s}=2 s+1$. If we take $\beta(=s)=$ $0 ; 1 / 2 ; 1 ; 3 / 2 ; 2 ; \ldots$ We will have $d+1=1 ; 2 ; 3 ; 4 ; 5 ; \ldots$

Note that as we invent $\Psi$ as a real field, we ought to take another normalization

$$
\int d^{d} x|\Psi|^{2}=1
$$

for the solutions of the motion equation. This case extra values of the parameter $\beta$ is possible, $\beta>d / 2$.

\subsection{Measurement of the space-time dimension inside hadrons}

We can take a renormdynamic scheme were $\Psi(g)$ is running coupling constant. The variable $z$ is a formation length and has dimension -1, RD equation for $\Psi$ in $\varphi_{D}^{3}$ model is

$$
\begin{gathered}
z \frac{d}{d z} \Psi=\frac{6-D}{2} \Psi+\gamma \Psi^{2} \\
\beta=\frac{D-6}{2}
\end{gathered}
$$

For high values of $\mathrm{z}, \beta=9$, so $D=24$. This value of $\mathrm{D}$ corresponds to the physical (transverse) degrees of freedom of the relativistic string, to the dimension of the external space in which this relativistic string lives. This is also the number of the quark - lepton matter degrees of freedom, $3 \cdot 6+6$. So, in these high energy reactions we measured the dimension of the space-time and matter and find the values predicted by relativistic string and SM. For lower energies, in this model, $D$ monotonically decrees until $D=6$, than the model (may) change form on the $\varphi_{D}^{4}, \beta=D-4$. So we have two scenarios of behavior. In one of them the dimension of the space-time inside hadrons has value 6 and higher. In another the dimension is 4 and higher.

Perturbative QCD indicates that we have a fixed point of RD in dimension slightly higher than 4 , and ordinary to hadron phase transition corresponds to the dimensional phase transition from slightly lower than 4, in QED, to slightly higher than 4 dimension in QCD. 


\subsection{General scalar field}

In general scalar field model $\varphi_{D}^{n}$,

$$
\beta=-d_{g}=\frac{n D}{2}-n-D .
$$

For $\varphi^{3}$ model, $\beta=9$ corresponds to $D=24$.

\subsection{0 $O(N)$-sigma model}

In tha case of the $O(N)$-sigma model

$$
\beta=D-2,
$$

For the experimental value of $\beta=9$, we have the dimension of the $M$-theory, $D=11$ !

\section{References}

[ALICE, 2010] K. Aamodt et al. [ALICE collaboration] Eur. Phys. J. C65 (2010) 111 [arXiv:1004.3034], [arXiv:1004.3514].

[Adler, 1965] S. L. Adler, Phys. Rev. 140 (1965) B736.

[Adler, Dashen, 1965] S.L. Adler, R. Dashen, Current Algebra and Applications to Particle Physics, Benjamin, New York 1965.

[Berry, Keating, 1997] M. Berry, Speculations on the Riemann operator, in proceedings of Symposium on Supersymmetry and Trace formulae, Cambridge 1997.

[Bogoliubov, Shirkov, 1959] N.N. Bogoliubov and D.V. Shirkov, Introduction to the Theory of Quantized Fields, New York 1959.

[Bogolubsky et al, 2009] I.L. Bogolubsky, E.M. Ilgenfritz, M. Müller-Preussker, A. Sternbeck, Phys.Lett.B 676 (2009) 69.

[Brekke, Freund, 1993] L. Brekke, P.G.O. Freund, Phys. Rep. 233 (1993) 1.

[Collins, 1984] J.C. Collins, Renormalization, Cambrige Univ. Press, London 1984.

[Darbaidze et al, 1978] Ya.Z. Darbaidze, N.V. Makhaldiani, A.N. Sisakian, L.A. Slepchenko, TMF 34 (1978) 303.

[Davis, 1977] M. Davis, Applied nonstandard analysis, New York 1977.

[Di Giacomo, Paffuti, Rossi, 1992] A. Di Giacomo, G. Paffuti, P. Rossi, Selected Problems in Theoretical Physics [With Solutions], World Scientific 1992.

[Drell, Hearn, 1966] S. D. Drell, A. C. Hearn, Exact Sum Rule For Nucleon Magnetic Moments, Phys. Rev. Lett. 16 (1966) 908.

[Ernst, Schmit, 1976] W. Ernst, I. Schmitt, Nuovo Cim. A33 (1976) 195.

[Faddeev, Takhtajan, 1987] L.D. Faddeev and L.A. Takhtajan, Hamiltonian methods in the theory of solitons, Springer, Berlin 1987.

[Ferrara, Porrati, Telegdi, 1992] S. Ferrara, M. Porrati, V.L. Telegdi, Phys. Rev. D, $g=2$ as the natural value of the tree level gyromagnetic ratio of elementary particles, 46 (1992) 3529. 
[Gasper, Rahman, 1990] G. Gasper, M. Rahman, Basic Hypergeometric Series, Cambridge Univ. Press, Cambridge 1990.

[Gerasimov, 1965] S.B. Gerasimov, A Sum Rule for Magnetic Moments and Damping of the Nucleon Magnetic Moment in Nuclei, J.Nucl.Phys.(USSR) 2 (1965) 598.

[Goldberger, Treiman, 1958] M.L. Goldberger and S.B. Treiman, Phys. Rev. 110 (1958) 1178.

[Gross, Wilczek, 1973] D.J. Gross, F. Wilczek, Phys. Rev. Lett. 30 (1973) 1343.

[Kaku, 2000] M. Kaku, Strings, Conformal Fields, and M-Theory, Springer, New York 2000.

[Kazakov et al, 1974] D.I. Kazakov, L.R. Lomidze, N.V. Makhaldiani, A.A. Vladimirov, Ultraviolet Asymptotics in Renormalizable Scalar Theories, in Communications of JINR, E2-8085, Dubna 1974.

[Kazakov, 2004] D.I. Kazakov, Supersymmetric Generalization of the Standard Model of Fundamental Interactions, Textbook, JINR Dubna 2004).

[Kazakov, Shirkov, 1980] D.I. Kazakov, D.V. Shirkov, Fortschr. d. Phys. 28 (1980) 447.

[Koba et al, 1972] Z. Koba, H.B. Nielsen, P. Olesen, Nucl. Phys. B40 (1972) 317.

[Koblitz, 1977] Neal Koblitz, p-adic numbers, p-adic analysis, and Zeta-functions, Springer-Verlag, New York 1977.

[Lomidze, 1994] I. Lomidze, On Some Generalizations of the Vandermonde Matrix and their Relations with the Eiler Beta-function, Georgian Math. Journal 1 (1994) 405.

[Makhaldiani, 1980] N.V. Makhaldiani, Approximate methods of the field theory and their applications in physics of high energy, condensed matter, plasma and hydrodynamics, Dubna 1980.

[Makhaldiani, 1986] N.M. Makhaldiani, Computational Quantum Field Theory, in Communication of JINR, P2-86-849, Dubna 1986.

[Makhaldiani, 1988] N.V. Makhaldiani, Number Fields Dynamics and the Compactification of Space Problem in the Unified Theories of Fields and Strings, in Communications of the JINR P2-88-916 Dubna 1988.

[Makhaldiani, 2003] N. Makhaldiani, Adelic Universe and Cosmological Constant, in Communications of the JINR E2-2003-215 Dubna 2003 [arXiv: hep-th/0312291].

[Makhaldiani, 2007] N. Makhaldiani, Nambu-Poisson dynamics of superintegrable systems, Atomic Nuclei, 70 (2007) 564.

[Makhaldiani, 2008] N.V. Makhaldiani, Renormdynamics and Scaling Functions, in Proc. of the XIX International Baldin Seminar on High Energy Physics Problems eds. A.N.Sissakian, V.V.Burov, A.I.Malakhov, S.G.Bondartenko, E.B.Plekhanov, Vol.II, p. 175, Dubna 2008.

[Makhaldiani, 2010] N.V. Makhaldiani, Renormdynamics, multiparticle production, negative binomial distribution and Riemann zeta function, [arXiv:1012.5939v1 [math-ph]] 24 Dec 2010.

[Makhaldiani, 2011] N. Makhaldiani, Fractal Calculus (H) and some Applications, Physics of Particles and Nuclei Letters, 8 (2011) 325.

[Matveev et al, 1976] V.A. Matveev, A.N. Sisakian, L.A. Slepchenko, Nucl. Phys. 23 (1976) 432.

[Miller, 1977] W. Miller, Jr. Symmetry and Separation of Variables, Addison-Wesley PC, London 1977.

[Moch, CALC 2012] S. Moch, QCD studies and Higgs searches at the LHC, Lectures at the International School-Workshop Calculations for Modern and Future Colliders, July23-August 2, Russian Federation, Dubna 2012. 
[Muehlleitner, CALC 2012] M.Muehlleitner, Composite Higgs and SUSY Physics at the LHC, Lectures at the International School-Workshop Calculations for Modern and Future Colliders, July23-August 2 , Russian Federation, Dubna 2012.

[Okun, 1982] L.B. Okun, Leptons and Quarks, North Holland 1982.

[Politzer, 1973] H.D. Politzer, Phys. Rev. Lett. 30 (1973) 1346.

[Pontryagin, 1983] L.S. Pontriagin et al., Mathematical Theory of Optimal Processes, Nauka, Moscow 1983.

['t Hooft, 1972] G. 't Hooft, report at the Marseille Conference on Yang-Mills Fields, 1972.

['t Hooft, 1972 2] G. 't Hooft, Nucl.Phys. B 61 (1973) 455.

[Smirnov, CALC 2012] V. Smirnov, Evaluating five-loop Konishi in N=4 SYM, Talk at the International School-Workshop Calculations for Modern and Future Colliders, July23-August 2, Russian Federation, Dubna 2012.

[Titchmarsh, 1986] E.C. Titchmarsh, The Theory of the Riemann zeta-function, Clarendon Press, Oxford 1986.

[Tokarev, Zborovsky, 2007] M.V. Tokarev, I. Zborovský, Z-Scaling in the Proton-Proton Collisions at RHIC, in Investigations of Properties of Nuclear Matter at High Temperature and Densities, Editid by A.N. Sisakian, F.A. Soifer, Dubna 2007.

[Treiman, Witten, Jackiw, Zumino, 1985] S. B. Treiman, E. Witten, R. Jackiw, B. Zumino, Current Algebra and Anomalies, World Scientific, Singapore 1985).

[Vladimirov, 1988] V.S. Vladimirov, Russian Math. Surveys 43 (1988) 19.

[Voloshin, Ter-Martyrosian, 1984] M.B. Voloshin, K.A. Ter-Martyrosian, Gauge Theory of Elementary Particles, Atomizdat, Moscow 1984.

[Watkins] M. Watkins at http://secamlocal.ex.ac.uk/ mwatkins/zeta/physics.htm.

[Weinberg, 1972] S. Weinberg, Gravitation and Cosmology, New York 1972.

[Weinberg, 1995] S. Weinberg, The Quantum Theory of Fields, Volum I - Foundations, Cambridge Univ. Press 1995.

[Weinberg, 1996] S. Weinberg, The Quantum Theory of Fields, Volum II - Modern Applications, Cambridge Univ. Press 1996.

[Weinberg, 2000] S. Weinberg, The Quantum Theory of Fields, Volum III - Supersymmetry, Cambridge Univ. Press 2000.

[Weisberger, 1966] W. I. Weisberger, Phys. Rev. 143 (1966) 1302. 